\title{
Local Risk-Minimization under Transaction Costs
}

\author{
Damien Lamberton, Huyên Pham* \\ Équipe d'Analyse et de Mathématiques Appliquées \\ Université de Marne-la-Vallée \\ 2, rue de la Butte Verte \\ F - 93166 Noisy-le-Grand Cedex \\ France \\ and \\ Martin Schweizer** \\ Technische Universität Berlin \\ Fachbereich Mathematik, MA 7-4 \\ Straße des 17. Juni 136 \\ D - 10623 Berlin \\ Germany
}

(to appear in Mathematics of Operations Research)

* This author is also affiliated to CREST, Laboratoire de Finance-Assurance, Paris.

** Research for this paper was partially carried out within Sonderforschungsbereich 373. This paper was printed using funds made available by the Deutsche Forschungsgemeinschaft. 
Abstract: We propose a new approach to the pricing and hedging of contingent claims under transaction costs in a general incomplete market in discrete time. Under the assumptions of a bounded mean-variance tradeoff, substantial risk and a nondegeneracy condition on the conditional variances of asset returns, we prove the existence of a locally risk-minimizing strategy inclusive of transaction costs for every square-integrable contingent claim. Then we show that local riskminimization is robust under the inclusion of transaction costs: The preceding strategy which is locally risk-minimizing inclusive of transaction costs in a model with bid-ask spreads on the underlying asset is also locally risk-minimizing without transaction costs in a fictitious model which is frictionless and where the fictitious asset price lies between the bid and ask price processes of the original model. In particular, our results apply to any nondegenerate model with a finite state space if the transaction cost parameter is sufficiently small.

Key words: option pricing, hedging, transaction costs, locally risk-minimizing strategies, mean-variance tradeoff

AMS 1991 subject classification: $90 \mathrm{~A} 09,60 \mathrm{~K} 30$

JEL Classification Numbers: G10, C60 


\section{Introduction}

This paper proposes a new approach to the pricing and hedging of contingent claims in the presence of transaction costs in a general incomplete market in discrete time. The existing literature on this topic can be separated into four major strands. Group zero studies the very basic question of characterizing arbitrage-free models with transaction costs; see for instance Jouini/Kallal (1995) or Ortu (1996). The first group of those really concerned with pricing and hedging considers self-financing strategies which exactly duplicate the desired payoff at maturity; examples are the papers by Merton (1989), Shen (1991) or Boyle/Vorst (1992). Quite apart from the fact that most contingent claims in an incomplete market will not permit the construction of such a strategy, it was also pointed out by Bensaid/Lesne/Pagès/Scheinkman (1992) that it can be less expensive to dominate the required payoff rather than to match it exactly. This super-replication approach was studied for instance by Bensaid/Lesne/Pagès/Scheinkman (1992), Edirisinghe/Naik/Uppal (1993) and Koehl/Pham/Touzi (1996) in discrete time or by Cvitanić/Karatzas (1996) in continuous time; a convergence result in this context has been obtained by Kusuoka (1995). As shown by Soner/Shreve/Cvitanić (1995), however, prices derived by super-replication are typically much too high and thus unfeasible in practice. A third major method for pricing options in the presence of transaction costs therefore explicitly introduces preferences, usually in the form of utility functions, to obtain a valuation formula; proponents of this approach are for instance Hodges/Neuberger (1989), Davis/Panas/Zariphopoulou (1993) or Constantinides/Zariphopoulou (1996).

The present paper is also to some degree in the spirit of the third methodology, but there are some differences. Like Leland (1985), Lott (1993), Henrotte (1993), Ahn/Dayal/Grannan/ Swindle (1995) or Kabanov/Safarian (1997), we do not insist on the use of self-financing strategies. Besides exact replication of the desired payoff at maturity, our optimality criterion for strategies is local risk-minimization, a local quadratic criterion first introduced by Schweizer (1988). For frictionless models without transaction costs, this approach has been studied and used by several authors. In the case of transaction cost models, it has been applied by Mercurio/Vorst (1997), but under rather restrictive assumptions and without completely rigorous proofs. Our first main result is the existence of a locally risk-minimizing strategy for every square-integrable contingent claim under certain technical but intuitive assumptions on the price process of our basic asset. The second main result is a very appealing economic interpretation of this strategy in terms of a model without transaction costs.

A quadratic risk-minimization approach can of course be criticized from a financial viewpoint since it gives equal weight to upside and downside risks. On the other hand, it has several properties which make its use rather appealing for practical purposes. One major advantage is its mathematical tractability which even leads to computable formulae in simple cases. On the theoretical side, it provides one possible way of selecting a pricing measure by specifying an optimality criterion; see the financial introduction of Delbaen/Monat/Schachermayer/Schweizer/Stricker (1997) for a discussion of this. On the practical side, it seems to produce hedging strategies whose initial costs are substantially lower than those of superreplicating strategies and whose replicating errors are relatively small; this is for instance illustrated by the numerical results of Mercurio/Vorst (1997).

The paper is structured as follows. Section 1 introduces our model, defines locally riskminimizing strategies and characterizes them in a way amenable to subsequent analysis. Section 2 defines two properties of an asset price process or more precisely its increments: boundedness of the mean-variance tradeoff process which was already introduced in Schweizer 
(1995) or (implicitly) in Schweizer (1988), and the condition of substantial risk which we believe is new. A number of technical results then lays the ground for section 3 where we first prove in Theorem 6 the existence of a locally risk-minimizing strategy inclusive of transaction costs under the assumptions of a bounded mean-variance tradeoff, substantial risk and a nondegeneracy condition on the conditional variances of asset returns. This is a general result which requires only square-integrability of the contingent claim to be hedged; no convexity or concavity is required. Theorem 7 then gives a striking economic interpretation of this strategy. In fact, we show that there exists a fictitious asset price process (depending on the contingent claim under consideration) which lies between the bid and ask price processes for our basic asset and which has the following property: if we hedge our contingent claim by a locally risk-minimizing strategy without transaction costs in this fictitious model, we obtain exactly the locally risk-minimizing strategy inclusive of transaction costs in the original model with bid-ask spreads. This means that the criterion of local risk-minimization has a rather remarkable robustness property under the inclusion of transaction costs. Section 4 discusses special cases and examples; we show in particular that our results apply to any nondegenerate model with a finite state space if the transaction cost parameter is small enough. Finally, the appendix contains a technical result on the minimization of a conditional variance which is used in the proof of Theorem 6 in section 3.

\section{Formulation of the problem}

This preliminary section has two purposes. We first introduce some terminology to formulate the basic problem that we study in this paper. Then we provide two auxiliary results which will help us later on to solve our optimization problem.

Let $(\Omega, \mathcal{F}, P)$ be a probability space with a filtration $\mathbb{F}=\left(\mathcal{F}_{k}\right)_{k=0,1, \ldots, T}$ for some fixed time horizon $T \in \mathbb{N}$. The stochastic process $X=\left(X_{k}\right)_{k=0,1, \ldots, T}$ describes the discounted price of some risky asset, here called stock, and so we assume that $X$ is adapted to $\mathbb{F}$ and nonnegative. There is also a riskless asset (called bond) whose discounted price is 1 at all times.

Remark. By considering discounted prices, we leave aside all problems related to the choice of a numeraire. These questions typically arise in the context of foreign exchange markets and would lead to additional modelling problems, including possibly transaction costs on the numeraire itself. This would certainly be an important extension of our approach, but is beyond the scope of the present paper.

Definition. For any stochastic process $Y=\left(Y_{k}\right)_{k=0,1, \ldots, T}$, we denote by $\Theta(Y)$ the space of all predictable processes $\vartheta=\left(\vartheta_{k}\right)_{k=1, \ldots, T+1}$ such that $\vartheta_{k} \Delta Y_{k} \in \mathcal{L}^{2}(P)$ for $k=1, \ldots, T$, where $\Delta Y_{k}:=Y_{k}-Y_{k-1}$. Recall that $\vartheta$ is predictable if and only if $\vartheta_{k}$ is $\mathcal{F}_{k-1}$-measurable for $k=1, \ldots, T+1$.

Definition. A (trading) strategy $\varphi$ is a pair of processes $\vartheta, \eta$ such that

$$
\begin{aligned}
& \vartheta \in \Theta(X), \\
& \eta=\left(\eta_{k}\right)_{k=0,1, \ldots, T} \text { is adapted }
\end{aligned}
$$

and 


$$
V_{k}(\varphi):=\vartheta_{k+1} X_{k}+\eta_{k} \in \mathcal{L}^{2}(P) \quad \text { for } k=0,1, \ldots, T
$$

The adapted process $V(\varphi)$ is then called the value process of $\varphi$.

To motivate the subsequent definition of the cost process of a strategy under transaction costs, it is helpful to first provide an interpretation of a strategy and to explain how it is implemented in our model. At each date $k$, one can choose the number $\vartheta_{k+1}$ of shares of stock and the number $\eta_{k}$ of bonds that one will hold until the following date $k+1$. Predictability of $\vartheta$ is imposed to obtain a simple correspondence to a formulation in continuous time. Clearly, $V_{k}(\varphi)$ is then the theoretical or book value of the portfolio $\left(\vartheta_{k+1}, \eta_{k}\right)$ with which one leaves date $k$ after trading. While the present notation conforms with the one in Schäl (1994) or Mercurio/Vorst (1997), it is different from the one used in Schweizer (1988, 1995), and we shall comment on this later on.

We view $V(\varphi)$ as a theoretical value because it is based on $X$ which itself is only a theoretical stock price. Stock transactions do not take place at this value; due to the presence of proportional transaction costs, they will involve a proportional bid-ask spread. More precisely, fix some transaction cost parameter $\lambda \in[0,1)$ and let $(1-\lambda) X_{k}$ and $(1+\lambda) X_{k}$ denote the bid and ask prices, respectively, for one share of stock at date $k$. Using the strategy $\varphi$ dictates at a given date $k$ to buy or sell (depending on the signs) $\eta_{k}-\eta_{k-1}$ bonds and $\vartheta_{k+1}-\vartheta_{k}$ shares of stock. The total outlay at date $k$ due to this transaction is therefore

$$
\begin{aligned}
& \eta_{k}-\eta_{k-1}+\left(\vartheta_{k+1}-\vartheta_{k}\right) X_{k}\left(1+\lambda \operatorname{sign}\left(\vartheta_{k+1}-\vartheta_{k}\right)\right) \\
& =V_{k}(\varphi)-V_{k-1}(\varphi)-\vartheta_{k}\left(X_{k}-X_{k-1}\right)+\lambda X_{k}\left|\vartheta_{k+1}-\vartheta_{k}\right|
\end{aligned}
$$

and summing over all dates up to $k$ yields the cumulative costs of the strategy $\varphi$. We denote here and in the following by sign the sign function with the convention

$$
\operatorname{sign}(0)=0
$$

Definition. The cost process of a strategy $\varphi=(\vartheta, \eta)$ is

$$
C_{k}(\varphi):=V_{k}(\varphi)-\sum_{j=1}^{k} \vartheta_{j} \Delta X_{j}+\lambda \sum_{j=1}^{k} X_{j}\left|\Delta \vartheta_{j+1}\right| \quad \text { for } k=0,1, \ldots, T
$$

Remarks. 1) More generally, we could model bid and ask prices by $\left(1-\lambda_{k}\right) X_{k}$ and $\left(1+\mu_{k}\right) X_{k}$ for some predictable processes $\lambda$ and $\mu$ with values in $[0,1)$ and $[0, \infty)$, respectively. But to simplify the notation, we have chosen a fixed and symmetric bid-ask spread.

2) Our next definition tacitly assumes that the cost process of any strategy is squareintegrable. If $\Omega$ is finite or if there are no transaction costs so that $\lambda=0$, this is clearly satisfied due to (1.1) and (1.2); in general, it will be a consequence of the technical assumptions imposed on $X$ in section 2 .

Definition. The risk process of a strategy $\varphi$ is

$$
R_{k}(\varphi):=E\left[\left(C_{T}(\varphi)-C_{k}(\varphi)\right)^{2} \mid \mathcal{F}_{k}\right] \quad \text { for } k=0,1, \ldots, T
$$


Definition. A contingent claim is a pair $\left(\bar{\vartheta}_{T+1}, \bar{\eta}_{T}\right)$ of $\mathcal{F}_{T}$-measurable random variables satisfying

$$
\bar{\vartheta}_{T+1} X_{T} \in \mathcal{L}^{2}(P)
$$

and

$$
H:=\bar{\vartheta}_{T+1} X_{T}+\bar{\eta}_{T} \in \mathcal{L}^{2}(P) .
$$

Intuitively, a contingent claim models a financial contract like for instance a call option. The quantities $\bar{\vartheta}_{T+1}$ and $\bar{\eta}_{T}$ describe the number of shares and bonds, respectively, that we need to have at the terminal date $T$ in order to fulfil our obligations; $H$ in (1.5) is then the (theoretical) value of this portfolio. In agreement with most of the existing literature, we assume for simplicity that there are no transaction costs for the liquidation of a position at date $T$ so that the sign of $\bar{\vartheta}_{T+1}$ is irrelevant for the computation of the value $H$. Explicit examples for contingent claims as well as various possible modes of settlement for an option will be discussed in section 4 .

Our goal in the following is to find for a given contingent claim a strategy which minimizes the risk in a local sense. To understand the idea underlying the subsequent definition, note that by (1.3), the cost difference $C_{T}(\varphi)-C_{k}(\varphi)$ and hence the risk $R_{k}(\varphi)$ depends on the strategy $\varphi$ via the variables $\eta_{k}, \eta_{k+1}, \ldots, \eta_{T}$ and $\vartheta_{k+1}, \vartheta_{k+2}, \ldots, \vartheta_{T+1}$. But the only decision we have to take at date $k$ is the choice of $\eta_{k}$ and $\vartheta_{k+1}$, and so we shall minimize $R_{k}(\varphi)$ only with respect to $\vartheta_{k+1}$ and $\eta_{k}$, leaving all other parameters fixed.

Definition. Let $\varphi=(\vartheta, \eta)$ be a strategy and $k \in\{0,1, \ldots, T-1\}$. A local perturbation of $\varphi$ at date $k$ is a strategy $\varphi^{\prime}=\left(\vartheta^{\prime}, \eta^{\prime}\right)$ with

and

$$
\vartheta_{j}^{\prime}=\vartheta_{j} \quad \text { for } j \neq k+1
$$

$$
\eta_{j}^{\prime}=\eta_{j} \quad \text { for } j \neq k \text {. }
$$

$\varphi$ is called locally risk-minimizing (inclusive of transaction costs) if we have

$$
R_{k}(\varphi) \leq R_{k}\left(\varphi^{\prime}\right) \quad P \text {-a.s. }
$$

for any date $k \in\{0,1, \ldots, T-1\}$ and any local perturbation $\varphi^{\prime}$ of $\varphi$ at date $k$.

The basic problem we study in this paper is then the following:

Given a contingent claim $\left(\bar{\vartheta}_{T+1}, \bar{\eta}_{T}\right)$, find a locally risk-minimizing strategy $\varphi=(\vartheta, \eta)$ with $\vartheta_{T+1}=\bar{\vartheta}_{T+1}$ and $\eta_{T}=\bar{\eta}_{T}$.

Remarks. 1) Note that date $T$ is excluded in the definition of local risk-minimization. Any local perturbation $\varphi^{\prime}$ of $\varphi$ must therefore satisfy the same terminal conditions as $\varphi$, i.e., $\vartheta_{T+1}^{\prime}=\vartheta_{T+1}$ and $\eta_{T}^{\prime}=\eta_{T}$, and so the preceding optimization problem makes sense as a formulation of hedging under transaction costs.

2) Since our definitions of a contingent claim and of the value process $V(\varphi)$ are different from the ones in Schweizer (1988), it is not completely evident that the preceding notion of local risk-minimization reduces to the one in Schweizer (1988) for $\lambda=0$. But we shall see in subsection 4.1 that this is indeed the case. 
Lemma 1. If $\varphi$ is locally risk-minimizing, then $C(\varphi)$ is a martingale and therefore

$$
R_{k}(\varphi)=E\left[R_{k+1}(\varphi) \mid \mathcal{F}_{k}\right]+\operatorname{Var}\left[\Delta C_{k+1}(\varphi) \mid \mathcal{F}_{k}\right] \quad P \text {-a.s. for } k=0,1, \ldots, T-1
$$

Proof. (1.6) is an immediate consequence of the martingale property of $C(\varphi)$ which in turn follows from a fairly standard argument. In fact, fix a date $k \in\{0,1, \ldots, T-1\}$ and define a pair $\varphi^{\prime}=\left(\vartheta^{\prime}, \eta^{\prime}\right)$ by setting $\vartheta^{\prime}:=\vartheta, \eta_{j}^{\prime}:=\eta_{j}$ for $j \neq k$ and

$$
\eta_{k}^{\prime}:=E\left[C_{T}(\varphi)-C_{k}(\varphi) \mid \mathcal{F}_{k}\right]+\eta_{k}
$$

Then $\eta^{\prime}$ is clearly adapted, and

$$
V_{k}\left(\varphi^{\prime}\right)=V_{k}(\varphi)+E\left[C_{T}(\varphi)-C_{k}(\varphi) \mid \mathcal{F}_{k}\right]
$$

shows that $\varphi^{\prime}$ satisfies (1.2) and therefore is a strategy, hence a local perturbation of $\varphi$ at date $k$. (Recall our tacit assumption that $C(\varphi)$ is square-integrable; see Lemma 3 below.) Due to (1.7) and the definition of $\varphi^{\prime}$, we have

$$
C_{T}\left(\varphi^{\prime}\right)-C_{k}\left(\varphi^{\prime}\right)=C_{T}(\varphi)-C_{k}(\varphi)-E\left[C_{T}(\varphi)-C_{k}(\varphi) \mid \mathcal{F}_{k}\right]
$$

hence

$$
R_{k}\left(\varphi^{\prime}\right)=\operatorname{Var}\left[C_{T}(\varphi)-C_{k}(\varphi) \mid \mathcal{F}_{k}\right] \leq E\left[\left(C_{T}(\varphi)-C_{k}(\varphi)\right)^{2} \mid \mathcal{F}_{k}\right]=R_{k}(\varphi)
$$

But because $\varphi$ is locally risk-minimizing, we must have equality $P$-a.s. and therefore $E\left[C_{T}(\varphi)-C_{k}(\varphi) \mid \mathcal{F}_{k}\right]=0$ P-a.s.

q.e.d.

Since $R_{k+1}(\varphi)$ does not depend on $\vartheta_{k+1}$ and $\eta_{k}$, Lemma 1 and (1.6) suggest to look for a locally risk-minimizing strategy by recursively minimizing $\operatorname{Var}\left[\Delta C_{k+1}(\varphi) \mid \mathcal{F}_{k}\right]$ with respect to $\vartheta_{k+1}$ and then determining $\eta_{k}$ from the martingale property of $C(\varphi)$. The next result tells us that this approach does indeed work.

Proposition 2. A strategy $\varphi=(\vartheta, \eta)$ is locally risk-minimizing if and only if it has the following two properties:

1) $C(\varphi)$ is a martingale.

2) For each $k \in\{0,1, \ldots, T-1\}, \vartheta_{k+1}$ minimizes

$$
\operatorname{Var}\left[V_{k+1}(\varphi)-\vartheta_{k+1}^{\prime} \Delta X_{k+1}+\lambda X_{k+1}\left|\vartheta_{k+2}-\vartheta_{k+1}^{\prime}\right| \mid \mathcal{F}_{k}\right]
$$

over all $\mathcal{F}_{k}$-measurable random variables $\vartheta_{k+1}^{\prime}$ such that $\vartheta_{k+1}^{\prime} \Delta X_{k+1} \in \mathcal{L}^{2}(P)$ and $\vartheta_{k+1}^{\prime} X_{k+1} \in \mathcal{L}^{2}(P)$.

Proof. By Lemma 1,C( $\varphi)$ is a martingale if $\varphi$ is locally risk-minimizing. If $C(\varphi)$ is a martingale, then (1.6) and the definition of $C(\varphi)$ imply that

$$
R_{k}(\varphi)=E\left[R_{k+1}(\varphi) \mid \mathcal{F}_{k}\right]+\operatorname{Var}\left[V_{k+1}(\varphi)-\vartheta_{k+1} \Delta X_{k+1}+\lambda X_{k+1}\left|\vartheta_{k+2}-\vartheta_{k+1}\right| \mid \mathcal{F}_{k}\right]
$$


by omitting $\mathcal{F}_{k}$-measurable terms from the conditional variance. Finally, $\vartheta_{k+1} \Delta X_{k+1} \in$ $\mathcal{L}^{2}(P)$ by (1.1), and the square-integrability of $\vartheta_{k+1} X_{k+1}$ will be a consequence of the technical assumptions imposed on $X$ in section 2 ; see Lemma 3 below.

Now fix $k \in\{0,1, \ldots, T-1\}$ and let $\varphi^{\prime}$ be a local perturbation of $\varphi$ at date $k$. Then

$$
C_{T}\left(\varphi^{\prime}\right)-C_{k+1}\left(\varphi^{\prime}\right)=C_{T}(\varphi)-C_{k+1}(\varphi)
$$

by the definition of the cost process, and since we may always assume that $C(\varphi)$ is a martingale, we obtain

$$
R_{k}\left(\varphi^{\prime}\right)=E\left[R_{k+1}(\varphi) \mid \mathcal{F}_{k}\right]+E\left[\left(\Delta C_{k+1}\left(\varphi^{\prime}\right)\right)^{2} \mid \mathcal{F}_{k}\right]
$$

by first conditioning on $\mathcal{F}_{k+1}$.

Suppose now first that 1) and 2) hold. Since $\varphi^{\prime}$ is a local perturbation of $\varphi$ at date $k$, we have

$$
V_{k+1}\left(\varphi^{\prime}\right)=V_{k+1}(\varphi) \quad \text { and } \quad \vartheta_{k+2}^{\prime}=\vartheta_{k+2}
$$

and therefore

$$
\Delta C_{k+1}\left(\varphi^{\prime}\right)=V_{k+1}(\varphi)-V_{k}\left(\varphi^{\prime}\right)-\vartheta_{k+1}^{\prime} \Delta X_{k+1}+\lambda X_{k+1}\left|\vartheta_{k+2}-\vartheta_{k+1}^{\prime}\right|
$$

Using (1.9), we obtain

$$
\begin{aligned}
R_{k}\left(\varphi^{\prime}\right) & \geq E\left[R_{k+1}(\varphi) \mid \mathcal{F}_{k}\right]+\operatorname{Var}\left[\Delta C_{k+1}\left(\varphi^{\prime}\right) \mid \mathcal{F}_{k}\right] \\
& \geq E\left[R_{k+1}(\varphi) \mid \mathcal{F}_{k}\right]+\operatorname{Var}\left[\Delta C_{k+1}(\varphi) \mid \mathcal{F}_{k}\right] \\
& =R_{k}(\varphi)
\end{aligned}
$$

where the second inequality uses 2), (1.8) and the irrelevance of $\mathcal{F}_{k}$-measurable terms in the conditional variance, and the last equality comes from (1.6). This shows that $\varphi$ is locally risk-minimizing.

Conversely, suppose that $\varphi$ is locally risk-minimizing so that 1) holds by Lemma 1 . Comparing (1.6) and (1.9) then shows that

$$
E\left[\left(\Delta C_{k+1}\left(\varphi^{\prime}\right)\right)^{2} \mid \mathcal{F}_{k}\right] \geq \operatorname{Var}\left[\Delta C_{k+1}(\varphi) \mid \mathcal{F}_{k}\right]
$$

for any $\mathcal{F}_{k}$-measurable choice of $\vartheta_{k+1}^{\prime}$ and $\eta_{k}^{\prime}$. In particular, we can fix $\vartheta_{k+1}^{\prime}$ and choose $\eta_{k}^{\prime}$ in such a way that $E\left[\Delta C_{k+1}\left(\varphi^{\prime}\right) \mid \mathcal{F}_{k}\right]=0$. Combining this with the preceding inequality and using the definition of $\Delta C_{k+1}\left(\varphi^{\prime}\right)$ and (1.10), we then obtain 2).

q.e.d.

According to the previous result, we can construct a locally risk-minimizing strategy by first recursively solving the optimization problem in part 2) of Proposition 2 backward through time and by then using the martingale property of $C(\varphi)$ to determine $\eta$. We shall use exactly this approach to prove the existence of a locally risk-minimizing strategy in section 3. 


\section{Conditions on $X$ and technical results}

This section collects a number of auxiliary results on integrability properties of strategies that we shall use in the next section to establish the existence of a locally risk-minimizing strategy. The processes $X^{\gamma}$ introduced below will play an important role in that construction. Let us signal at this point that for a non-degenerate finite tree model, the results of this section will hold trivially; this is explained more carefully in subsection 4.2. Apart from the next definition and relation (2.3), this section can therefore be skipped by those readers who are only interested in the case of a finite probability space.

Throughout this section, we shall assume that $X$ is a square-integrable process so that $X_{k} \in \mathcal{L}^{2}(P)$ for $k=0,1, \ldots, T$.

Definition. We denote by $\Gamma$ the class of all adapted processes $\gamma=\left(\gamma_{k}\right)_{k=0,1, \ldots, T}$ with values in $[-1,+1]$. For $\gamma \in \Gamma$, the process $X^{\gamma}$ is defined by

$$
X_{k}^{\gamma}:=X_{k}\left(1+\lambda \gamma_{k}\right) \quad \text { for } k=0,1, \ldots, T \text {. }
$$

If $\varphi=(\vartheta, \eta)$ is a strategy, the process $V^{\gamma}(\varphi)$ is defined by

$$
V_{k}^{\gamma}(\varphi):=\vartheta_{k+1} X_{k}^{\gamma}+\eta_{k} \quad \text { for } k=0,1, \ldots, T
$$

Since $\lambda \in[0,1)$, it is clear that each process $X^{\gamma}$ is again nonnegative, adapted and squareintegrable. The significance of the processes $X^{\gamma}$ is explained by a result of Jouini/Kallal (1995). They show that the pair of processes $X^{-1}=(1-\lambda) X$ and $X^{+1}=(1+\lambda) X$ defines an arbitrage-free system of bid and ask prices if and only if there exist a process $\gamma \in \Gamma$ and a probability measure $Q$ equivalent to $P$ such that $X^{\gamma}$ is a $Q$-martingale; see also Koehl/Pham/Touzi (1996). Intuitively, such a $Q$ can be interpreted as a price system which is compatible with bid and ask prices given by $X^{-1}$ and $X^{+1}$, respectively. $X^{\gamma}$ is then a kind of re-valuation of the stock, and $V^{\gamma}(\varphi)$ is of course the value process of the strategy $\varphi$ if one works in units of $X^{\gamma}$ instead of $X$.

Our main goal in this section is to show that under suitable conditions on $X$ and $\lambda$, we have $\Theta\left(X^{\gamma}\right)=\Theta(X)$ for all $\gamma \in \Gamma$. Roughly speaking, this means that the same strategies can be used for all reasonable choices of units.

Definition. We say that $X$ has substantial risk if there is a constant $c<\infty$ such that

$$
\frac{X_{k-1}^{2}}{E\left[\Delta X_{k}^{2} \mid \mathcal{F}_{k-1}\right]} \leq c \quad P \text {-a.s. for } k=1, \ldots, T .
$$

The smallest constant $c$ satisfying $(2.2)$ will be denoted by $c_{\mathrm{SR}}$.

The condition of substantial risk essentially provides a lower bound on the conditional variances of the increments of $X$, but it also has a very intuitive interpretation. If we define the return process $\varrho$ of $X$ as usual by

$$
X_{k}=X_{k-1}\left(1+\varrho_{k}\right) \quad \text { for } k=1, \ldots, T \text {, }
$$


then (2.2) can equivalently be written as

$$
E\left[\varrho_{k}^{2} \mid \mathcal{F}_{k-1}\right] \geq \frac{1}{c}>0 \quad P \text {-a.s. for } k=1, \ldots, T
$$

In particular, this means that $X$ has substantial risk if and only if we have some lower bound on the returns of $X$. A simple example is the case where each $\varrho_{k}$ is independent of $\mathcal{F}_{k-1}$ ("independent returns", if $\mathbb{F}$ is generated by $X$ ) and not identically 0. Condition (2.2) is also satisfied in every non-degenerate finite tree model; this is discussed in more detail in subsection 4.2 .

Lemma 3. Assume that $X$ has substantial risk. Then:

1) $\Theta\left(X^{\gamma}\right) \supseteq \Theta(X)$ for every $\gamma \in \Gamma$.

2) $V_{k}^{\gamma}(\varphi) \in \mathcal{L}^{2}(P)$ for $k=0,1, \ldots, T$, for every $\gamma \in \Gamma$ and for every strategy $\varphi$.

3) $\vartheta_{k+1} X_{k} \in \mathcal{L}^{2}(P)$ for $k=0,1, \ldots, T$ and for every $\vartheta \in \Theta(X)$.

4) $C_{k}(\varphi) \in \mathcal{L}^{2}(P)$ for $k=0,1, \ldots, T$ and for every strategy $\varphi$.

Proof. By the definition of $X^{\gamma}$, we have

$$
\vartheta_{k} \Delta X_{k}^{\gamma}=\vartheta_{k} \Delta X_{k}+\lambda \gamma_{k} \vartheta_{k} \Delta X_{k}+\lambda \vartheta_{k} X_{k-1} \Delta \gamma_{k}
$$

and since $\gamma$ is bounded, 1) will follow from 3). By the definition of $V^{\gamma}(\varphi)$,

$$
V_{k}^{\gamma}(\varphi)=V_{k}(\varphi)+\lambda \gamma_{k} \vartheta_{k+1} X_{k}
$$

and so (1.2) shows that 2) will also follow from 3). The definition of $C(\varphi)$ implies the useful relation

$$
\begin{aligned}
\Delta C_{k}(\varphi) & =\Delta V_{k}(\varphi)-\vartheta_{k} \Delta X_{k}+\lambda X_{k}\left|\Delta \vartheta_{k+1}\right| \\
& =\vartheta_{k+1} X_{k}\left(1+\lambda \operatorname{sign}\left(\Delta \vartheta_{k+1}\right)\right)+\eta_{k}-\vartheta_{k} X_{k}\left(1+\lambda \operatorname{sign}\left(\Delta \vartheta_{k+1}\right)\right)-\eta_{k-1} \\
& =\vartheta_{k+1} X_{k}^{\gamma}+\eta_{k}-\vartheta_{k} X_{k}^{\gamma}-\eta_{k-1} \\
& =\Delta V_{k}^{\gamma}(\varphi)-\vartheta_{k} \Delta X_{k}^{\gamma},
\end{aligned}
$$

if we define the process $\gamma$ by $\gamma_{k}:=\operatorname{sign}\left(\Delta \vartheta_{k+1}\right)$ for $k=0,1, \ldots, T$. Note that $\gamma \in \Gamma$ because $\vartheta$ is predictable; (2.3) and (1.2) therefore show that 4) follows from 2) and 1), and so it only remains to prove 3$)$. But this is easy:

$$
E\left[\left(\vartheta_{k+1} X_{k}\right)^{2}\right]=E\left[\left(\vartheta_{k+1} \Delta X_{k+1}\right)^{2} \frac{X_{k}^{2}}{E\left[\Delta X_{k+1}^{2} \mid \mathcal{F}_{k}\right]}\right] \leq c_{\mathrm{SR}} E\left[\left(\vartheta_{k+1} \Delta X_{k+1}\right)^{2}\right]<\infty
$$

since $\vartheta \in \Theta(X)$ and $X$ has substantial risk.

q.e.d.

Remark. In particular, the assertions 3) and 4) in Lemma 3 clear up two points of integrability that were left open in section 1; see Lemma 1 and Proposition 2.

In order to obtain the reverse inclusion $\Theta\left(X^{\gamma}\right) \subseteq \Theta(X)$, we first study the mean-variance tradeoff process of $X^{\gamma}$. 
Definition. For $\gamma \in \Gamma$, the mean-variance tradeoff process of $X^{\gamma}$ is

$$
\widehat{K}_{\ell}^{\gamma}:=\sum_{j=1}^{\ell} \frac{\left(E\left[\Delta X_{j}^{\gamma} \mid \mathcal{F}_{j-1}\right]\right)^{2}}{\operatorname{Var}\left[\Delta X_{j}^{\gamma} \mid \mathcal{F}_{j-1}\right]} \quad \text { for } \ell=0,1, \ldots, T
$$

If $\widehat{K}^{\gamma}$ is $P$-a.s. bounded by a constant, we denote by $c_{\mathrm{MVT}}(\gamma)$ the smallest constant $c<\infty$ such that

$$
\Delta \widehat{K}_{\ell}^{\gamma}=\frac{\left(E\left[\Delta X_{\ell}^{\gamma} \mid \mathcal{F}_{\ell-1}\right]\right)^{2}}{\operatorname{Var}\left[\Delta X_{\ell}^{\gamma} \mid \mathcal{F}_{\ell-1}\right]} \leq c \quad P \text {-a.s. for } \ell=1, \ldots, T \text {. }
$$

One frequently made assumption on $X$ is that $X$ should have a bounded mean-variance tradeoff process; see for instance Schäl (1994) or Schweizer (1995). The following result provides a sufficient condition to ensure that $X^{\gamma}$ then also has a bounded mean-variance tradeoff.

Proposition 4. Assume that $X$ has a bounded mean-variance tradeoff and substantial risk. Fix $\gamma \in \Gamma$ and assume that there is a constant $c>0$ such that

$$
\operatorname{Var}\left[\Delta X_{k}^{\gamma} \mid \mathcal{F}_{k-1}\right] \geq c \operatorname{Var}\left[\Delta X_{k} \mid \mathcal{F}_{k-1}\right] \quad P \text {-a.s. for } k=1, \ldots, T
$$

Then $X^{\gamma}$ has a bounded mean-variance tradeoff, and $\Theta\left(X^{\gamma}\right)=\Theta(X)$.

Proof. We first show that (2.5) implies that $X^{\gamma}$ has a bounded mean-variance tradeoff. According to (2.4), this will be true if

$$
\left(E\left[\Delta X_{k}^{\gamma} \mid \mathcal{F}_{k-1}\right]\right)^{2} \leq \text { const. } \operatorname{Var}\left[\Delta X_{k} \mid \mathcal{F}_{k-1}\right] \quad P \text {-a.s. for } k=1, \ldots, T \text {. }
$$

But since

$$
\Delta X_{k}^{\gamma}=\Delta X_{k}+\lambda \gamma_{k} X_{k}-\lambda \gamma_{k-1} X_{k-1}=\Delta X_{k}+\lambda \gamma_{k} \Delta X_{k}+\lambda X_{k-1} \Delta \gamma_{k},
$$

we even have

$$
\begin{aligned}
E\left[\left(\Delta X_{k}^{\gamma}\right)^{2} \mid \mathcal{F}_{k-1}\right] & \leq 2(1+\lambda)^{2} E\left[\Delta X_{k}^{2} \mid \mathcal{F}_{k-1}\right]+8 \lambda^{2} X_{k-1}^{2} \\
& \leq \text { const. } E\left[\Delta X_{k}^{2} \mid \mathcal{F}_{k-1}\right] \\
& \leq \text { const. }\left(1+c_{\mathrm{MVT}}(0)\right) \operatorname{Var}\left[\Delta X_{k} \mid \mathcal{F}_{k-1}\right]
\end{aligned}
$$

where we use first that $\gamma \in \Gamma$ is bounded by 1 , then (2.2) and finally (2.4) with $\gamma \equiv 0$.

Since the inclusion $\Theta(X) \subseteq \Theta\left(X^{\gamma}\right)$ was already established in Lemma 3, it only remains to show that $\Theta\left(X^{\gamma}\right) \subseteq \Theta(X)$. To that end, let $X^{\gamma}=X_{0}^{\gamma}+M^{\gamma}+A^{\gamma}$ be the Doob decomposition of $X^{\gamma}$ so that

$$
\vartheta_{k} \Delta X_{k}^{\gamma}=\vartheta_{k} \Delta M_{k}^{\gamma}+\vartheta_{k} \Delta A_{k}^{\gamma}=\vartheta_{k} \Delta M_{k}^{\gamma}+\vartheta_{k} E\left[\Delta X_{k}^{\gamma} \mid \mathcal{F}_{k-1}\right]
$$

and

$$
\operatorname{Var}\left[\Delta X_{k}^{\gamma} \mid \mathcal{F}_{k-1}\right]=E\left[\left(\Delta M_{k}^{\gamma}\right)^{2} \mid \mathcal{F}_{k-1}\right]
$$


Since $X^{\gamma}$ has a bounded mean-variance tradeoff, (2.4) shows that $\vartheta \in \Theta\left(X^{\gamma}\right)$ if and only if $\vartheta_{k} \Delta M_{k}^{\gamma} \in \mathcal{L}^{2}(P)$ for $k=1, \ldots, T$ for which we shortly write $\vartheta \in L^{2}\left(M^{\gamma}\right)$. The same holds of course for $X=X^{0}$. Now if $\vartheta$ is predictable and (2.5) holds, then

$$
E\left[\left(\vartheta_{k} \Delta M_{k}\right)^{2} \mid \mathcal{F}_{k-1}\right]=\vartheta_{k}^{2} \operatorname{Var}\left[\Delta X_{k} \mid \mathcal{F}_{k-1}\right] \leq \frac{1}{c} \vartheta_{k}^{2} \operatorname{Var}\left[\Delta X_{k}^{\gamma} \mid \mathcal{F}_{k-1}\right]=\frac{1}{c} E\left[\left(\vartheta_{k} \Delta M_{k}^{\gamma}\right)^{2} \mid \mathcal{F}_{k-1}\right]
$$

implies that $L^{2}\left(M^{\gamma}\right) \subseteq L^{2}(M)$, hence $\Theta\left(X^{\gamma}\right) \subseteq \Theta(X)$ since both mean-variance tradeoffs are bounded, and this completes the proof.

q.e.d.

Proposition 4 is quite satisfactory if one knows enough about $\gamma$ to establish the estimate (2.5). But since we shall usually not be in such a position, we next show how to impose conditions on $X$ and $\lambda$ which ensure that (2.5) holds uniformly over all $\gamma \in \Gamma$.

Proposition 5. If there is a constant $\delta<1$ such that

$$
2 \lambda \sqrt{\frac{E\left[X_{k}^{2} \mid \mathcal{F}_{k-1}\right]}{\operatorname{Var}\left[\Delta X_{k} \mid \mathcal{F}_{k-1}\right]}} \leq \delta \quad P \text {-a.s. for } k=1, \ldots, T,
$$

then (2.5) holds simultaneously for all $\gamma \in \Gamma$, with $c=1-\delta$. In particular, (2.6) holds if $X$ has a bounded mean-variance tradeoff and substantial risk and if $\lambda$ satisfies

$$
4 \lambda^{2}\left(1+2 c_{\mathrm{MVT}}(0)+2 c_{\mathrm{SR}}\left(1+c_{\mathrm{MVT}}(0)\right)\right)<1
$$

Proof. Since $X_{k}^{\gamma}=X_{k}\left(1+\lambda \gamma_{k}\right)$, omitting $\mathcal{F}_{k-1}$-measurable terms from the conditional variance yields

$$
\begin{aligned}
\operatorname{Var}\left[\Delta X_{k}^{\gamma} \mid \mathcal{F}_{k-1}\right] & =\operatorname{Var}\left[\Delta X_{k}+\lambda \gamma_{k} X_{k} \mid \mathcal{F}_{k-1}\right] \\
& \geq \operatorname{Var}\left[\Delta X_{k} \mid \mathcal{F}_{k-1}\right]-2 \lambda \sqrt{\operatorname{Var}\left[\Delta X_{k} \mid \mathcal{F}_{k-1}\right] \operatorname{Var}\left[\gamma_{k} X_{k} \mid \mathcal{F}_{k-1}\right]}
\end{aligned}
$$

by the Cauchy-Schwarz inequality. Since $\gamma$ is bounded by $1,(2.6)$ implies that

$$
\operatorname{Var}\left[\gamma_{k} X_{k} \mid \mathcal{F}_{k-1}\right] \leq E\left[\gamma_{k}^{2} X_{k}^{2} \mid \mathcal{F}_{k-1}\right] \leq \frac{\delta^{2}}{4 \lambda^{2}} \operatorname{Var}\left[\Delta X_{k} \mid \mathcal{F}_{k-1}\right]
$$

hence (2.5) with $c=1-\delta$. To obtain (2.6) from (2.7), write

$$
E\left[X_{k}^{2} \mid \mathcal{F}_{k-1}\right]=\operatorname{Var}\left[\Delta X_{k} \mid \mathcal{F}_{k-1}\right]+\left(X_{k-1}+E\left[\Delta X_{k} \mid \mathcal{F}_{k-1}\right]\right)^{2}
$$

and use the estimates

$$
X_{k-1}^{2} \leq c_{\mathrm{SR}} E\left[\Delta X_{k}^{2} \mid \mathcal{F}_{k-1}\right]
$$

from (2.2) and

$$
E\left[\Delta X_{k}^{2} \mid \mathcal{F}_{k-1}\right] \leq \operatorname{Var}\left[\Delta X_{k} \mid \mathcal{F}_{k-1}\right]\left(1+c_{\mathrm{MVT}}(0)\right)
$$

from (2.4).

q.e.d.

Condition (2.6) should be viewed as a quantitative formulation of the assumption that transaction costs have to be sufficiently small for our subsequent results to hold. This kind of condition is well known from the existing literature on transaction cost problems in discrete time, and we shall comment below on its relation to other work in this area. 


\section{Existence and structure of an optimal strategy}

This section proves the existence of a locally risk-minimizing strategy under transaction costs and describes its structure in more detail. The basic idea to derive an optimal strategy is quite simple: we just solve for each $k$ the conditional variance minimization problem in part 2 ) of Proposition 2 to obtain the optimal $\vartheta$-component. The existence of a predictable process $\vartheta$ which minimizes the relevant conditional variance almost surely at each step is rather easily established from a general result proved in the appendix. But since this only gives existence by means of a measurable choice, it is not clear if the resulting $\vartheta$ is sufficiently integrable, i.e., if it lies in the space $\Theta(X)$. We therefore provide at the same time a representation of the optimal $\vartheta$ which allows us to prove the required integrability. If $\Omega$ is finite, integrability is of course no problem, but the expression for $\vartheta$ will still be of interest in view of the interpretation it will provide later on. Moreover, there are very natural situations where $\Omega$ is not finite; one example is the process obtained by discretizing the Black-Scholes model in time as in subsection 4.5 .

Throughout this section, we assume that $X$ is a square-integrable process.

Theorem 6. Assume that $X$ has a bounded mean-variance tradeoff, substantial risk and satisfies (2.6) as well as

$$
\operatorname{Var}\left[\Delta X_{k} \mid \mathcal{F}_{k-1}\right]>0 \quad P \text {-a.s. for } k=1, \ldots, T
$$

Then for any contingent claim $\left(\bar{\vartheta}_{T+1}, \bar{\eta}_{T}\right)$, there exists a locally risk-minimizing strategy $\varphi^{*}=\left(\vartheta^{*}, \eta^{*}\right)$ with $\vartheta_{T+1}^{*}=\bar{\vartheta}_{T+1}$ and $\eta_{T}^{*}=\bar{\eta}_{T}$. Its first component $\vartheta^{*}$ can be described as follows: There exists a process $\delta^{*} \in \Gamma$ such that if we define $\nu \in \Gamma$ by setting $\nu_{0}:=0$ and

$$
\nu_{k}:=\operatorname{sign}\left(\vartheta_{k+1}^{*}-\vartheta_{k}^{*}\right)+\delta_{k}^{*} I_{\left\{\vartheta_{k+1}^{*}=\vartheta_{k}^{*}\right\}} \quad \text { for } k=1, \ldots, T
$$

then we have

$$
\vartheta_{k}^{*}=\frac{\operatorname{Cov}\left(\Delta V_{k}^{\nu}\left(\varphi^{*}\right), \Delta X_{k}^{\nu} \mid \mathcal{F}_{k-1}\right)}{\operatorname{Var}\left[\Delta X_{k}^{\nu} \mid \mathcal{F}_{k-1}\right]} \quad P \text {-a.s. for } k=1, \ldots, T \text {. }
$$

Proof. This is essentially just a backward induction argument relying on the existence results from the appendix and the technical results from section 2 ; the main difficulty is to write it down as concisely as possible. We shall prove by backward induction the existence of a predictable process $\vartheta^{*}$ with $\vartheta_{T+1}^{*}=\bar{\vartheta}_{T+1}$ and satisfying assertions a), b) below for $k=0,1, \ldots, T$ and $\mathrm{c}), \mathrm{d}), \mathrm{e})$ for $k=1, \ldots, T$ :

a) $\vartheta_{k+1}^{*} X_{k} \in \mathcal{L}^{2}(P)$.

b) $\quad W_{k}^{*}:=H-\sum_{j=k+1}^{T} \vartheta_{j}^{*} \Delta X_{j}+\lambda \sum_{j=k+1}^{T} X_{j}\left|\Delta \vartheta_{j+1}^{*}\right| \in \mathcal{L}^{2}(P)$.

c) There exists an $\mathcal{F}_{k-1}$-measurable random variable $\delta_{k}^{*}$ with values in $[-1,+1]$ such that if we define $\nu_{k}$ by (3.1), then we have

$$
\vartheta_{k}^{*}=\frac{\operatorname{Cov}\left(E\left[W_{k}^{*} \mid \mathcal{F}_{k}\right]+\lambda \nu_{k} X_{k} \vartheta_{k+1}^{*}, X_{k}\left(1+\lambda \nu_{k}\right) \mid \mathcal{F}_{k-1}\right)}{\operatorname{Var}\left[X_{k}\left(1+\lambda \nu_{k}\right) \mid \mathcal{F}_{k-1}\right]} \quad P \text {-a.s. }
$$


d) $\vartheta_{k}^{*} \Delta X_{k} \in \mathcal{L}^{2}(P)$.

e) $\vartheta_{k}^{*}$ minimizes

$$
\operatorname{Var}\left[E\left[W_{k}^{*} \mid \mathcal{F}_{k}\right]-\vartheta_{k} \Delta X_{k}+\lambda X_{k}\left|\vartheta_{k+1}^{*}-\vartheta_{k}\right| \mid \mathcal{F}_{k-1}\right]
$$

over all $\mathcal{F}_{k-1}$-measurable random variables $\vartheta_{k}$ satisfying $\vartheta_{k} \Delta X_{k} \in \mathcal{L}^{2}(P)$ and $\vartheta_{k} X_{k} \in \mathcal{L}^{2}(P)$.

Once all this is established, we define $\eta^{*}$ by

$$
\eta_{k}^{*}:=E\left[W_{k}^{*} \mid \mathcal{F}_{k}\right]-\vartheta_{k+1}^{*} X_{k} \quad \text { for } k=0,1, \ldots, T \text {. }
$$

Then $\eta^{*}$ is clearly adapted and $\vartheta_{k+1}^{*} X_{k}+\eta_{k}^{*} \in \mathcal{L}^{2}(P)$ by b) so that thanks to d), $\varphi^{*}=\left(\vartheta^{*}, \eta^{*}\right)$ will be a strategy satisfying $\vartheta_{T+1}^{*}=\bar{\vartheta}_{T+1}$ and $\eta_{T}^{*}=\bar{\eta}_{T}$. By the definitions of $\eta^{*}$ and $W_{k}^{*}$, $V_{k}\left(\varphi^{*}\right)=E\left[W_{k}^{*} \mid \mathcal{F}_{k}\right]$ for all $k$ and $C\left(\varphi^{*}\right)$ is a martingale. Hence we conclude from e) and Proposition 2 that $\varphi^{*}$ is locally risk-minimizing. Moreover, the definition of $\eta^{*}$ implies that

$$
E\left[W_{k}^{*} \mid \mathcal{F}_{k}\right]+\lambda \nu_{k} X_{k} \vartheta_{k+1}^{*}=V_{k}^{\nu}\left(\varphi^{*}\right),
$$

and so (3.2) is just a restatement of (3.3).

To complete the proof, it thus remains to establish a) - e). If we define $\vartheta_{T+1}^{*}:=\bar{\vartheta}_{T+1}$, it is clear from (1.4) and (1.5) that a) and b) hold for $k=T$. We shall show that the validity of a) and b) for any $k$ implies the existence of an $\mathcal{F}_{k-1}$-measurable random variable $\vartheta_{k}^{*}$ satisfying c) - e) for $k$, and that this in turn implies the validity of a) and b) for $k-1$.

So assume that a) and b) hold for $k$. Set

$$
\begin{aligned}
& \overline{\operatorname{sign}}(x):=\operatorname{sign}(x)+I_{\{x=0\}}:=\left\{\begin{array}{ll}
+1 & \text { for } x \geq 0 \\
-1 & \text { for } x<0
\end{array},\right. \\
& \underline{\operatorname{sign}}(x):=\operatorname{sign}(x)-I_{\{x=0\}}:=\left\{\begin{array}{ll}
+1 & \text { for } x>0 \\
-1 & \text { for } x \leq 0
\end{array},\right.
\end{aligned}
$$

and define the functions

$$
f_{k}(c, \omega):=\operatorname{Var}\left[E\left[W_{k}^{*} \mid \mathcal{F}_{k}\right]-c X_{k}+\lambda X_{k}\left|\vartheta_{k+1}^{*}-c\right| \mid \mathcal{F}_{k-1}\right](\omega)
$$

and

$$
\begin{aligned}
g_{k}(c, \alpha, \omega):= & \operatorname{Cov}\left(E\left[W_{k}^{*} \mid \mathcal{F}_{k}\right]+\lambda X_{k} \vartheta_{k+1}^{*} S_{k}^{(\alpha, c)}, X_{k}\left(1+\lambda S_{k}^{(\alpha, c)}\right) \mid \mathcal{F}_{k-1}\right)(\omega) \\
& -c \operatorname{Var}\left[X_{k}\left(1+\lambda S_{k}^{(\alpha, c)}\right) \mid \mathcal{F}_{k-1}\right](\omega)
\end{aligned}
$$

with

$$
S_{k}^{(\alpha, c)}:=\alpha \overline{\operatorname{sign}}\left(\vartheta_{k+1}^{*}-c\right)+(1-\alpha) \underline{\operatorname{sign}}\left(\vartheta_{k+1}^{*}-c\right),
$$

where the conditional variances and covariances are all computed with respect to a regular conditional distribution of $\left(E\left[W_{k}^{*} \mid \mathcal{F}_{k}\right], X_{k}, \vartheta_{k+1}^{*}\right)$ given $\mathcal{F}_{k-1}$. From Propositions A2 and A3 in the appendix, we then obtain the existence of an $\mathcal{F}_{k-1}$-measurable random variable $\vartheta_{k}^{*}$ and an $\mathcal{F}_{k-1}$-measurable random variable $\alpha_{k}^{*}$ with values in $[0,1]$ such that

$$
f_{k}\left(\vartheta_{k}^{*}(\omega), \omega\right) \leq f_{k}(c, \omega) \quad P \text {-a.s. for all } c
$$


and

$$
g_{k}\left(\vartheta_{k}^{*}(\omega), \alpha_{k}^{*}(\omega), \omega\right)=0 \quad P \text {-a.s. }
$$

If we define $\delta_{k}^{*}:=2 \alpha_{k}^{*}-1$, then we get

$$
S_{k}^{\left(\alpha_{k}^{*}, \vartheta_{k}^{*}\right)}=\operatorname{sign}\left(\vartheta_{k+1}^{*}-\vartheta_{k}^{*}\right)+\delta_{k}^{*} I_{\left\{\vartheta_{k+1}^{*}=\vartheta_{k}^{*}\right\}}=\nu_{k}
$$

and therefore (3.3) by rewriting (3.6) so that c) holds for $k$. Note that the ratio in (3.3) is well-defined thanks to a), b) for $k$ and the boundedness of $\nu_{k}$.

Next we prove that $\mathrm{d}$ ) holds for $k$. Let $\gamma$ be any process in $\Gamma$ with $\gamma_{k}=\nu_{k}$ and define

$$
W_{k}^{\gamma}:=E\left[W_{k}^{*} \mid \mathcal{F}_{k}\right]+\lambda \nu_{k} X_{k} \vartheta_{k+1}^{*} .
$$

By a) and b) for $k, W_{k}^{\gamma} \in \mathcal{L}^{2}(P)$, and so (3.3) can be rewritten as

$$
\vartheta_{k}^{*}=\frac{\operatorname{Cov}\left(W_{k}^{\gamma}, \Delta X_{k}^{\gamma} \mid \mathcal{F}_{k-1}\right)}{\operatorname{Var}\left[\Delta X_{k}^{\gamma} \mid \mathcal{F}_{k-1}\right]}
$$

since $\mathcal{F}_{k-1}$-measurable terms do not matter for the conditional variance and covariance. The Cauchy-Schwarz inequality and Proposition 5 then imply that

$$
\begin{aligned}
E\left[\left(\vartheta_{k}^{*} \Delta X_{k}\right)^{2}\right] & \leq E\left[\frac{\operatorname{Var}\left[W_{k}^{\gamma} \mid \mathcal{F}_{k-1}\right]}{\operatorname{Var}\left[\Delta X_{k}^{\gamma} \mid \mathcal{F}_{k-1}\right]} E\left[\Delta X_{k}^{2} \mid \mathcal{F}_{k-1}\right]\right] \\
& \leq \frac{1}{c} E\left[E\left[\left(W_{k}^{\gamma}\right)^{2} \mid \mathcal{F}_{k-1}\right] \frac{E\left[\Delta X_{k}^{2} \mid \mathcal{F}_{k-1}\right]}{\operatorname{Var}\left[\Delta X_{k} \mid \mathcal{F}_{k-1}\right]}\right] \\
& \leq \frac{1}{c}\left(1+c_{\mathrm{MVT}}(0)\right) E\left[\left(W_{k}^{\gamma}\right)^{2}\right]<\infty
\end{aligned}
$$

so that d) holds for $k$. Since $X$ has substantial risk, we conclude as in the proof of Lemma 3 that $\vartheta_{k}^{*} X_{k-1} \in \mathcal{L}^{2}(P)$ which establishes a) for $k-1$. At the same time, we obtain $\vartheta_{k}^{*} X_{k}=$ $\vartheta_{k}^{*} \Delta X_{k}+\vartheta_{k}^{*} X_{k-1} \in \mathcal{L}^{2}(P)$ as required in e).

The validity of e) for $k$ is now almost immediate. In fact, if $\vartheta_{k}$ is $\mathcal{F}_{k-1}$-measurable and satisfies $\vartheta_{k} \Delta X_{k} \in \mathcal{L}^{2}(P)$ and $\vartheta_{k} X_{k} \in \mathcal{L}^{2}(P)$, then we have

$$
\operatorname{Var}\left[E\left[W_{k}^{*} \mid \mathcal{F}_{k}\right]-\vartheta_{k} \Delta X_{k}+\lambda X_{k}\left|\vartheta_{k+1}^{*}-\vartheta_{k}\right| \mid \mathcal{F}_{k-1}\right](\omega)=f_{k}\left(\vartheta_{k}(\omega), \omega\right) \quad P \text {-a.s. }
$$

and so e) for $k$ follows from (3.5). Finally,

$$
W_{k-1}^{*}=W_{k}^{*}-\vartheta_{k}^{*} \Delta X_{k}+\lambda X_{k}\left|\vartheta_{k+1}^{*}-\vartheta_{k}^{*}\right| \in \mathcal{L}^{2}(P)
$$

due to b) for $k$, d) for $k$, a) for $k$ and the square-integrability of $\vartheta_{k}^{*} X_{k}$. Thus b) holds for $k-1$, and this completes the induction.

q.e.d.

From an economic point of view, the next result is now the central contribution of this paper. It provides a striking interpretation of the strategy $\varphi^{*}$ which can be viewed as a robustness result for the approach of local risk-minimization. To formulate this, let us first 
point out that we could or should have been more precise in our terminology by calling the strategy $\varphi^{*}$ in Theorem 6 "locally risk-minimizing for the price process $X$ inclusive of transaction costs". In the same way, we can talk of a strategy which is "locally risk-minimizing for a price process $Z$ without transaction costs" if we replace $X$ by $Z$ and set $\lambda=0$ in all the definitions in section 1 .

Theorem 7. Assume the conditions of Theorem 6. The strategy $\varphi^{*}$ which is locally riskminimizing for the price process $X$ inclusive of transaction costs is then also the strategy which is locally risk-minimizing for the price process $X^{\nu}$ without transaction costs, where $\nu$ is given by (3.1).

Proof. Let $\varphi=(\vartheta, \eta)$ be locally risk-minimizing for the price process $X^{\nu}$ without transaction costs and denote by $\widetilde{V}_{k}(\varphi):=\vartheta_{k+1} X_{k}^{\nu}+\eta_{k}$ and

$$
\widetilde{C}_{k}(\varphi):=\widetilde{V}_{k}(\varphi)-\sum_{j=1}^{k} \vartheta_{j} \Delta X_{j}^{\nu}
$$

the corresponding value and cost processes, when the price process is $X^{\nu}$. Since $X^{\nu}$ has a bounded mean-variance tradeoff by Proposition 5, the results of Schweizer (1988) then imply that $\widetilde{C}(\varphi)$ is a martingale and that

$$
\vartheta_{k}=\frac{\operatorname{Cov}\left(\Delta \tilde{V}_{k}(\varphi), \Delta X_{k}^{\nu} \mid \mathcal{F}_{k-1}\right)}{\operatorname{Var}\left[\Delta X_{k}^{\nu} \mid \mathcal{F}_{k-1}\right]} \quad P \text {-a.s. for } k=1, \ldots, T \text {. }
$$

(Actually, this is not quite true as it stands: since the value process is defined differently in Schweizer (1988), we also have to show that the resulting locally risk-minimizing strategy is the same as with the present definition of $\widetilde{V}(\varphi)$. This will be done in subsection 4.1.) By the definition of $V^{\nu}(\varphi)$ in (2.1), we have $\widetilde{V}(\varphi)=V^{\nu}(\varphi)$, and so (3.2) shows that $\vartheta$ and $\vartheta^{*}$ coincide. Moreover, $\widetilde{C}(\varphi)=C(\varphi)$ by $(2.3)$, and since $\varphi$ is mean-self-financing according to Lemma I.7 of Schweizer (1988), $\widetilde{C}(\varphi)=C(\varphi)$ is a martingale. By Lemma 1 , so is $C\left(\varphi^{*}\right)$, and since both have the same terminal value

$$
H-\sum_{j=1}^{T} \vartheta_{j} \Delta X_{j}^{\nu}=H-\sum_{j=1}^{T} \vartheta_{j}^{*} \Delta X_{j}^{\nu}
$$

$C(\varphi)$ and $C\left(\varphi^{*}\right)$ must coincide, hence also $\eta$ and $\eta^{*}$.

q.e.d.

Theorem 7 shows that the criterion of local risk-minimization possesses a remarkable kind of robustness property under the addition of transaction costs. In fact, it tells us that we can construct a strategy which is locally risk-minimizing for $X$ inclusive of transaction costs by first re-valuing the stock at a suitable price $X^{\nu}$ within the bid-ask range and then simply minimizing the risk locally for transaction cost free prices set at $X^{\nu}$. This stability property of local risk-minimization complements results of Prigent (1995) and Runggaldier/Schweizer (1995) on stability under convergence and can thus be viewed as yet another argument in favour of this approach to the hedging of contingent claims. 
Remark. To put things into perspective, we should perhaps add here that Theorem 7 is primarily a theoretical structural result. To effect the transformation from $X$ to $X^{\nu}$, we have to know $\nu$ which is given by (3.1). But this raises two difficulties: to compute $\nu$, we have to know the optimal strategy $\vartheta^{*}$, and we also have to find $\delta^{*}$ which is in general only given by an existence result. We shall see below that - as in most transaction cost problems - it seems very difficult to obtain more explicit expressions for the optimal strategy $\varphi^{*}$.

Thanks to the identification in Theorem 7 , we can also say more about the value process of the optimal strategy $\varphi^{*}$. To do this, we first recall the notion of the minimal signed martingale measure $\widehat{P}^{\gamma}$ for $X^{\gamma}$, where $\gamma \in \Gamma$. Define the process $\widehat{Z}^{\gamma}$ by

$$
\begin{aligned}
\widehat{Z}_{k}^{\gamma} & :=\prod_{j=1}^{k}\left(1-\frac{E\left[\Delta X_{j}^{\gamma} \mid \mathcal{F}_{j-1}\right]}{\operatorname{Var}\left[\Delta X_{j}^{\gamma} \mid \mathcal{F}_{j-1}\right]}\left(\Delta X_{j}^{\gamma}-E\left[\Delta X_{j}^{\gamma} \mid \mathcal{F}_{j-1}\right]\right)\right) \\
& =\prod_{j=1}^{k}\left(1-\frac{\Delta A_{j}^{\gamma}}{E\left[\left(\Delta M_{j}^{\gamma}\right)^{2} \mid \mathcal{F}_{j-1}\right]} \Delta M_{j}^{\gamma}\right),
\end{aligned}
$$

where $X^{\gamma}=X_{0}^{\gamma}+M^{\gamma}+A^{\gamma}$ is the Doob decomposition of $X^{\gamma}$. If $X^{\gamma}$ has a bounded meanvariance tradeoff, it is not hard to check that $\widehat{Z}^{\gamma}$ is a square-integrable $P$-martingale starting at 1 and that $\widehat{Z}^{\gamma} X^{\gamma}$ is also a $P$-martingale; see for instance Schweizer (1995). We are therefore justified in calling the signed measure $\widehat{P}^{\gamma}$ defined by

$$
\frac{d \widehat{P}^{\gamma}}{d P}:=\widehat{Z}_{T}^{\gamma}
$$

a signed martingale measure for $X^{\gamma}$, and we can define conditional expectations under $\widehat{P}^{\gamma}$ via the Bayes rule by

$$
\widehat{E}^{\gamma}\left[U_{\ell} \mid \mathcal{F}_{k}\right]:=\frac{1}{\widehat{Z}_{k}^{\gamma}} E\left[U_{\ell} \widehat{Z}_{\ell}^{\gamma} \mid \mathcal{F}_{k}\right]
$$

for any $\mathcal{F}_{\ell}$-measurable random variable $U_{\ell} \in \mathcal{L}^{2}(P)$.

Corollary 8. Assume the conditions of Theorem 6 and let $\varphi^{*}$ be locally risk-minimizing inclusive of transaction costs for the contingent claim $\left(\bar{\vartheta}_{T+1}, \bar{\eta}_{T}\right)$. Then we have

$$
V_{k}^{\nu}\left(\varphi^{*}\right)=\widehat{E}^{\nu}\left[\bar{\vartheta}_{T+1} X_{T}^{\nu}+\bar{\eta}_{T} \mid \mathcal{F}_{k}\right] \quad P \text {-a.s. for } k=0,1, \ldots, T
$$

where $\nu$ is given by (3.1).

Proof. Thanks to Theorem 7, this is well known from the results of Schweizer $(1988,1995)$ on local risk-minimization without transaction costs, but for completeness we give a proof based on Theorem 6. Since $V_{T}^{\nu}\left(\varphi^{*}\right)=\bar{\vartheta}_{T+1} X_{T}^{\nu}+\bar{\eta}_{T}$, it is enough to show that $V^{\nu}\left(\varphi^{*}\right)$ is a $\widehat{P}^{\nu}$-martingale or more precisely that $\widehat{Z}^{\nu} V^{\nu}\left(\varphi^{*}\right)$ is a $P$-martingale. Since $\varphi^{*}$ is locally riskminimizing inclusive of transaction costs, we know from Lemma 1 and (2.3) that the cost process

$$
C\left(\varphi^{*}\right)=V^{\nu}\left(\varphi^{*}\right)-\sum \vartheta_{j}^{*} \Delta X_{j}^{\nu}
$$


is a $P$-martingale, and so plugging in (3.2) yields

$$
\begin{aligned}
0 & =\operatorname{Var}\left[\Delta X_{k}^{\nu} \mid \mathcal{F}_{k-1}\right] E\left[\Delta V_{k}^{\nu}\left(\varphi^{*}\right)-\vartheta_{k}^{*} \Delta X_{k}^{\nu} \mid \mathcal{F}_{k-1}\right] \\
& =E\left[\operatorname{Var}\left[\Delta X_{k}^{\nu} \mid \mathcal{F}_{k-1}\right] \Delta V_{k}^{\nu}\left(\varphi^{*}\right) \mid \mathcal{F}_{k-1}\right]-\operatorname{Cov}\left(\Delta V_{k}^{\nu}\left(\varphi^{*}\right), \Delta X_{k}^{\nu} \mid \mathcal{F}_{k-1}\right) E\left[\Delta X_{k}^{\nu} \mid \mathcal{F}_{k-1}\right] \\
& =E\left[\Delta V_{k}^{\nu}\left(\varphi^{*}\right)\left(\operatorname{Var}\left[\Delta X_{k}^{\nu} \mid \mathcal{F}_{k-1}\right]-E\left[\Delta X_{k}^{\nu} \mid \mathcal{F}_{k-1}\right]\left(\Delta X_{k}^{\nu}-E\left[\Delta X_{k}^{\nu} \mid \mathcal{F}_{k-1}\right]\right)\right) \mid \mathcal{F}_{k-1}\right] \\
& =\operatorname{Var}\left[\Delta X_{k}^{\nu} \mid \mathcal{F}_{k-1}\right] E\left[\Delta V_{k}^{\nu}\left(\varphi^{*}\right) \frac{\widehat{Z}_{k}^{\nu}}{\widehat{Z}_{k-1}^{\nu}} \mid \mathcal{F}_{k-1}\right]
\end{aligned}
$$

which proves the assertion.

q.e.d.

Like Theorem 7, Corollary 8 has a very appealing economic interpretation. It tells us that the value process of a locally risk-minimizing strategy under transaction costs is the conditional expectation of the terminal payoff to be hedged under a certain (signed) measure $\widehat{P}^{\nu}$. This $\widehat{P}^{\nu}$ has the property that it turns into a martingale one particular process (namely $X^{\nu}$ ) which lies between the bid and ask price processes for our stock. In the terminology of Jouini/Kallal (1995), we have therefore identified $\widehat{P}^{\nu}$ as that (generalized) price system consistent with transaction costs which corresponds to the criterion of local risk-minimization inclusive of transaction costs. We say "generalized" since $\widehat{P}^{\nu}$ is typically not equivalent to $P$, but only a signed measure.

\section{Special cases and examples}

\subsection{The case of no transaction costs}

Consider first the case where $\lambda=0$ so that there are no transaction costs. We show in this subsection that we then recover from Theorem 6 the results obtained in Schweizer (1988). This is not immediately evident since we use here slightly different definitions. Recall that in Schweizer (1988), the value and cost processes of a strategy $\varphi=(\vartheta, \eta)$ were defined as

$$
\widetilde{V}_{k}(\varphi):=\vartheta_{k} X_{k}+\eta_{k} \quad \text { for } k=0,1, \ldots, T
$$

with $\vartheta_{0}:=0$ and

$$
\widetilde{C}_{k}(\varphi):=\widetilde{V}_{k}(\varphi)-\sum_{j=1}^{k} \vartheta_{j} \Delta X_{j} \quad \text { for } k=0,1, \ldots, T
$$

and a contingent claim was simply an $\mathcal{F}_{T}$-measurable random variable $H \in \mathcal{L}^{2}(P)$. Under the assumption that $X$ has a bounded mean-variance tradeoff, a locally risk-minimizing strategy $\widetilde{\varphi}$ for $H$ was then characterized by the properties that $\widetilde{V}_{T}(\widetilde{\varphi})=H P$-a.s., $\widetilde{C}(\widetilde{\varphi})$ is a martingale and

$$
\widetilde{\vartheta}_{k}=\frac{\operatorname{Cov}\left(\Delta \widetilde{V}_{k}(\widetilde{\varphi}), \Delta X_{k} \mid \mathcal{F}_{k-1}\right)}{\operatorname{Var}\left[\Delta X_{k} \mid \mathcal{F}_{k-1}\right]} \quad P \text {-a.s. for } k=1, \ldots, T \text {. }
$$


Let us now show that for $\lambda=0$, the strategy $\varphi^{*}$ in Theorem 6 gives the same solution as the preceding $\widetilde{\varphi}$. More precisely, we do not have $\varphi^{*}=\widetilde{\varphi}$, but we claim that $\vartheta^{*}=\widetilde{\vartheta}$ and $V\left(\varphi^{*}\right)=\widetilde{V}(\widetilde{\varphi})$, provided of course that $H$ is given by $H=\bar{\vartheta}_{T+1} X_{T}+\bar{\eta}_{T}$. In fact, it is obvious that

$$
V_{T}\left(\varphi^{*}\right)=H=\widetilde{V}_{T}(\widetilde{\varphi}) \quad P \text {-a.s. }
$$

and therefore

$$
\vartheta_{T}^{*}=\frac{\operatorname{Cov}\left(H, \Delta X_{T} \mid \mathcal{F}_{T-1}\right)}{\operatorname{Var}\left[\Delta X_{T} \mid \mathcal{F}_{T-1}\right]}=\widetilde{\vartheta}_{T} \quad P \text {-a.s. }
$$

by (3.2) and (4.1), since $X^{\nu}=X$ for $\lambda=0$. If we already know that $V_{k+1}\left(\varphi^{*}\right)=\widetilde{V}_{k+1}(\widetilde{\varphi})$ and $\vartheta_{k+1}^{*}=\widetilde{\vartheta}_{k+1}$, the martingale property of $C\left(\varphi^{*}\right)$ and $\widetilde{C}(\widetilde{\varphi})$ gives

$$
E\left[\Delta V_{k+1}\left(\varphi^{*}\right) \mid \mathcal{F}_{k}\right]=\vartheta_{k+1}^{*} E\left[\Delta X_{k+1} \mid \mathcal{F}_{k}\right]=E\left[\Delta \widetilde{V}_{k+1}(\widetilde{\varphi}) \mid \mathcal{F}_{k}\right]
$$

and therefore $V_{k}\left(\varphi^{*}\right)=\widetilde{V}_{k}(\widetilde{\varphi}) P$-a.s. This implies in turn that $\vartheta_{k}^{*}=\widetilde{\vartheta}_{k}$ again by $(3.2)$ and (4.1), and so the assertion follows by backward induction.

Note that due to the differing definitions of $V(\varphi)$ and $\widetilde{V}(\varphi)$, we cannot have $\eta^{*}=\widetilde{\eta}$ in general. But for practical purposes, $\varphi^{*}$ and $\widetilde{\varphi}$ can clearly be viewed as equivalent. We remark that the preceding argument clears up a point that was left open in the proof of Theorem 7 .

\subsection{The case where $\Omega$ is finite}

For a finite probability space $\Omega$, the problem of finding a locally risk-minimizing strategy inclusive of transaction costs has also been studied by Mercurio/Vorst (1997). They also assert the existence of an optimal strategy, but their proof does not seem to be completely rigorous. Moreover, they do not obtain any expression for the optimal strategy.

So let $\Omega$ be finite and $X$ strictly positive. Our main point in this subsection is that in any non-degenerate finite event tree model, all the assumptions of Theorem 6 are satisfied as soon as the transaction cost parameter $\lambda$ is sufficiently small. A finite event tree model is defined by the properties that $\mathcal{F}_{0}=\{\emptyset, \Omega\}, \mathbb{F}$ is generated by $X$ and $\Omega$ is finite with $P[\{\omega\}]>0$ for all $\omega \in \Omega$. This implies that $X_{0}$ is deterministic and that the returns

$$
\varrho_{k}(\omega):=\frac{X_{k}(\omega)}{X_{k-1}(\omega)}-1
$$

can only take a finite number of values for each $\omega$ at each date $k$. The date-price pairs $\left(k, X_{k}(\omega)\right)$ can then be viewed as the nodes of a tree with root $\left(0, X_{0}\right)$ which completely describes $\mathbb{F}$ and $X$; this explains the terminology. A finite event tree model is called nondegenerate if each return variable $\varrho_{k}$ takes at least two distinct values. In the tree notation, this means that each node has at least two branches leaving from it, while in mathematical terms, it simply says that $\operatorname{Var}\left[\Delta X_{k} \mid \mathcal{F}_{k-1}\right](\omega)>0$ for all $k$ and $\omega$. Since $\Omega$ is finite, it is clear that $X$ has then substantial risk and a bounded mean-variance tradeoff.

Lemma 9. Let

$$
\varrho^{\min }:=\max _{k, \omega} \max \left\{Y \mid Y \text { is } \mathcal{F}_{k-1} \text {-measurable and } Y \leq \varrho_{k}\right\}
$$

and

$$
\varrho^{\max }:=\min _{k, \omega} \min \left\{Y \mid Y \text { is } \mathcal{F}_{k-1} \text {-measurable and } Y \geq \varrho_{k}\right\}
$$


If $\lambda$ satisfies

$$
\frac{1+\lambda}{1-\lambda}<\frac{1+\varrho^{\max }}{1+\varrho^{\min }}
$$

then (2.5) holds uniformly for all $\gamma \in \Gamma$.

Proof. Because $\Omega$ is finite, it is enough to show that $\operatorname{Var}\left[\Delta X_{k}^{\gamma} \mid \mathcal{F}_{k-1}\right](\omega)$ is bounded below uniformly in $\gamma \in \Gamma$ for each $k$ and each $\omega$. Since

$$
\operatorname{Var}\left[\Delta X_{k}^{\gamma} \mid \mathcal{F}_{k-1}\right]=\operatorname{Var}\left[\Delta X_{k}+\lambda \gamma_{k} X_{k} \mid \mathcal{F}_{k-1}\right]=X_{k-1}^{2} \operatorname{Var}\left[\varrho_{k}+\lambda \gamma_{k}\left(1+\varrho_{k}\right) \mid \mathcal{F}_{k-1}\right]
$$

and $X$ is strictly positive, it is sufficient to obtain a lower bound for

$$
\operatorname{Var}\left[\varrho_{k}+\lambda \gamma_{k}\left(1+\varrho_{k}\right) \mid \mathcal{F}_{k-1}\right](\omega)
$$

which is uniform in $\gamma$. If we define

$$
\begin{aligned}
\ell_{k-1} & :=\max \left\{Y \mid Y \text { is } \mathcal{F}_{k-1} \text {-measurable and } Y \leq \varrho_{k}\right\}, \\
u_{k-1} & :=\min \left\{Y \mid Y \text { is } \mathcal{F}_{k-1} \text {-measurable and } Y \geq \varrho_{k}\right\}
\end{aligned}
$$

then clearly the complement of $\left(\ell_{k-1}, u_{k-1}\right)$ will be hit by $\varrho_{k}$ with positive conditional probability given $\mathcal{F}_{k-1}$. By the definition of $\Gamma$, each $\gamma_{k}$ has values in $[-1,+1]$; for each $\gamma \in \Gamma$, the conditional probability given $\mathcal{F}_{k-1}$ that

$$
Z_{k}^{(\gamma)}:=\varrho_{k}+\lambda \gamma_{k}\left(1+\varrho_{k}\right)=\varrho_{k}\left(1+\lambda \gamma_{k}\right)+\lambda \gamma_{k}
$$

hits the complement of the $\mathcal{F}_{k-1}$-measurable interval

$$
I_{k-1}:=\left(\ell_{k-1}(1+\lambda)+\lambda, u_{k-1}(1-\lambda)-\lambda\right)
$$

is therefore also positive. If $I_{k-1}(\omega)$ is non-empty, $\operatorname{Var}\left[Z_{k}^{(\gamma)} \mid \mathcal{F}_{k-1}\right](\omega)$ must then be $>0$, and the lower bound will be uniform in $\gamma$ since $I_{k-1}$ does not involve $\gamma$. But a sufficient condition for $I_{k-1}(\omega) \neq \emptyset$ for all $k$ and all $\omega$ is obviously

$$
\left(\max _{k, \omega} \ell_{k-1}(\omega)\right)(1+\lambda)+\lambda<\left(\min _{k, \omega} u_{k-1}(\omega)\right)(1-\lambda)-\lambda
$$

and so (4.2) implies the desired assertion.

q.e.d.

By rewriting (4.2) as

$$
\lambda<\frac{\varrho^{\max }-\varrho^{\min }}{\varrho^{\max }+\varrho^{\min }+2},
$$

we thus conclude that for sufficiently small transaction costs, we can always find a locally risk-minimizing strategy if we have a non-degenerate finite event tree model. For a binomial model where each $\varrho_{k}$ takes values in $\{u, d\}$ only, (4.2) reduces to the condition

$$
\frac{1+\lambda}{1-\lambda}<\frac{1+u}{1+d}
$$


which also appears in Boyle/Vorst (1992) and Koehl/Pham/Touzi (1996); see the discussion in the next subsection.

\subsection{Attainable claims}

In this subsection, we briefly discuss those claims which can be perfectly hedged including transaction costs. For a more detailed study, we refer to Koehl/Pham/Touzi (1996).

Definition. A contingent claim $\left(\bar{\vartheta}_{T+1}, \bar{\eta}_{T}\right)$ is called attainable if there exists a strategy $\varphi=(\vartheta, \eta)$ with $\vartheta_{T+1}=\bar{\vartheta}_{T+1}, \eta_{T}=\bar{\eta}_{T}$ and such that the cost process $C(\varphi)$ is almost surely constant over time. As usual, $\varphi$ is then called self-financing (inclusive of transaction costs).

Clearly, a contingent claim is attainable if and only if it can be hedged by a locally riskminimizing strategy whose risk process is identically zero with probability one. In particular, this implies that the terminal value $H=\bar{\vartheta}_{T+1} X_{T}+\bar{\eta}_{T}$ can be written as

$$
H=H_{0}+\sum_{j=1}^{T} \vartheta_{j}^{H} \Delta X_{j}-\lambda \sum_{j=1}^{T} X_{j}\left|\Delta \vartheta_{j+1}^{H}\right| \quad P \text {-a.s. }
$$

for some $\vartheta^{H} \in \Theta(X)$; the optimal strategy is then $\varphi^{*}=\varphi=\left(\vartheta^{H}, \eta^{*}\right)$, and its value process is given by

$$
V_{k}\left(\varphi^{*}\right)=H_{0}+\sum_{j=1}^{k} \vartheta_{j}^{H} \Delta X_{j}-\lambda \sum_{j=1}^{k} X_{j}\left|\Delta \vartheta_{j+1}^{H}\right| \quad P \text {-a.s. for } k=0,1, \ldots, T \text {. }
$$

The problem of finding a self-financing strategy including transaction costs was first studied by Boyle/Vorst (1992) in a binomial model, where the returns $\varrho_{k}$ are i.i.d. with values in $\{u, d\}$. They showed by elementary calculations that European call and put options are attainable, that their values at time 0 are given by an expectation quite analogous to (3.7), and that the attaining self-financing strategies are unique. Their results were subsequently extended to arbitrary attainable claims in general (not necessarily finite) discrete-time models by Koehl/Pham/Touzi (1996) who showed in particular that a similar condition as (4.2) is sufficient for the uniqueness of the self-financing strategy. Moreover, Koehl/Pham/Touzi (1996) also proved that the price of $H$ at date 0 is given by $H_{0}$ and that it can be written as an expectation under a suitable measure $Q^{H}$ as in (3.7). Other related work in binomial models was done by Merton (1989), Shen (1991) and Shirakawa/Konno (1995), among others. However, all these papers did not use local risk-minimization.

\subsection{Settlement modes and uniqueness issues}

A natural question in our problem is the uniqueness of the optimal strategy. We have not been able to obtain a general result so far, but we can give at least some partial answers. Since uniqueness is closely related to the way that options are settled, we first briefly discuss the latter issue in the special case where the contingent claim under consideration is a European call option on $X$ with maturity $T$ and strike price $K$. For this contract, there are at least three different ways to specify the terminal condition $\left(\bar{\vartheta}_{T+1}, \bar{\eta}_{T}\right)$. Settlement with delivery means that upon exercise, the option writer has to hand over physically one share of stock 
in exchange for $K$ units of cash so that

$$
\begin{aligned}
\bar{\vartheta}_{T+1} & =I_{\left\{(1+\lambda) X_{T}>K\right\}}, \\
\bar{\eta}_{T} & =-K I_{\left\{(1+\lambda) X_{T}>K\right\}} .
\end{aligned}
$$

With cash settlement, the option writer has to pay out in cash the value of the option at date $T$. In this case, we have

$$
\begin{aligned}
\bar{\vartheta}_{T+1} & =0 \\
\bar{\eta}_{T} & =\left((1+\lambda) X_{T}-K\right)^{+}=\left((1+\lambda) X_{T}-K\right) I_{\left\{(1+\lambda) X_{T}>K\right\}} .
\end{aligned}
$$

Finally, settlement up to the seller means that the option writer can give the option holder any portfolio $\left(\bar{\vartheta}_{T+1}, \bar{\eta}_{T}\right)$ of his own choice as long as its value $H=\bar{\vartheta}_{T+1} X_{T}+\bar{\eta}_{T}$ is equal to the option's value $\left((1+\lambda) X_{T}-K\right)^{+}$.

For contingent claims which are attainable in the sense of the preceding subsection, the results of Koehl/Pham/Touzi (1996) show that an analogue of (4.2) always implies uniqueness of the corresponding optimal strategy. Provided that call options are attainable, this would guarantee uniqueness for both cash settlement and settlement with delivery. On the other hand, it is not surprising that there will never be uniqueness for the case of settlement up to the seller if transaction costs are different from 0 . To see this, take $K=0$ and consider first the simple buy-and-hold strategy $\varphi$ given by

$$
\begin{array}{ll}
\vartheta_{k}=1+\lambda & \text { for } k=1, \ldots, T+1, \\
\eta_{k}=0 & \text { for } k=0,1, \ldots, T .
\end{array}
$$

This strategy is clearly self-financing, hence locally risk-minimizing, and it has a terminal value of $(1+\lambda) X_{T}$. Its initial cost is $(1+\lambda) X_{0}$ if we neglect as usual transaction costs at date 0 . An alternative strategy $\varphi^{\prime}$ is given by

$$
\begin{aligned}
& \vartheta_{k}^{\prime}= \begin{cases}\frac{1+\lambda}{1-\lambda} & \text { for } k=1, \ldots, T \\
0 & \text { for } k=T+1,\end{cases} \\
& \eta_{k}^{\prime}= \begin{cases}0 & \text { for } k=0, \ldots, T-1 \\
(1+\lambda) X_{T} & \text { for } k=T .\end{cases}
\end{aligned}
$$

Clearly, this strategy also leads to a final value of $(1+\lambda) X_{T}$. Its only transaction is at date $T$ where it generates a cost increment of

$$
\begin{aligned}
\Delta C_{T}\left(\varphi^{\prime}\right) & =\Delta V_{T}\left(\varphi^{\prime}\right)-\vartheta_{T}^{\prime} \Delta X_{T}+\lambda X_{T}\left|\Delta \vartheta_{T+1}^{\prime}\right| \\
& =(1+\lambda) X_{T}-\frac{1+\lambda}{1-\lambda} X_{T-1}-\frac{1+\lambda}{1-\lambda} \Delta X_{T}+\lambda X_{T} \frac{1+\lambda}{1-\lambda} \\
& =0 .
\end{aligned}
$$

Hence $\varphi^{\prime}$ is also self-financing and therefore locally risk-minimizing. Its initial cost is $\frac{1+\lambda}{1-\lambda} X_{0}$, and it is obviously different from the first strategy. Since $\varphi^{\prime}$ is unambiguously more expensive, it is of course clear that one will discard it in favour of the buy-and-hold strategy $\varphi$. But our main point here is that the criterion of local risk-minimization alone may be insufficient to make such a distinction. 


\subsection{Explicit calculations in an example}

It is a familar feature of all models involving transaction costs that explicit expressions for option values or hedging strategies are rather difficult to obtain. Not surprisingly, this also happens in our present approach. To illustrate how far one can go, we consider in this subsection a model with i.i.d. returns where $\mathbb{F}$ is generated by $X$ and the returns $\varrho_{k}$ are i.i.d. random variables in $\mathcal{L}^{2}(P)$. This implies that $X_{k}=X_{k-1}\left(1+\varrho_{k}\right)$, where each $\varrho_{k}$ is independent of $\mathcal{F}_{k-1}$ and distributed like some fixed random variable $\varrho$. To have $X$ nonnegative and not constant, we assume that $\varrho \geq-1 P$-a.s. and that $\operatorname{Var}[\varrho]>0$. The mean-variance tradeoff process of $X$ is then given by

$$
\Delta \widehat{K}_{\ell}=\frac{\left(E\left[\Delta X_{\ell} \mid \mathcal{F}_{\ell-1}\right]\right)^{2}}{\operatorname{Var}\left[\Delta X_{\ell} \mid \mathcal{F}_{\ell-1}\right]}=\frac{(E[\varrho])^{2}}{\operatorname{Var}[\varrho]} \quad \text { for } \ell=1, \ldots, T
$$

it is clearly bounded, and

$$
c_{\mathrm{MVT}}(0)=\frac{(E[\varrho])^{2}}{\operatorname{Var}[\varrho]}
$$

Moreover,

$$
\frac{X_{k-1}^{2}}{E\left[\Delta X_{k}^{2} \mid \mathcal{F}_{k-1}\right]}=\frac{1}{E\left[\varrho^{2}\right]} \quad \text { for } k=1, \ldots, T,
$$

and so we see that $X$ has also substantial risk and

$$
c_{\mathrm{SR}}=\frac{1}{E\left[\varrho^{2}\right]}
$$

Finally, condition (2.6) takes the form

$$
\lambda \leq \frac{\delta}{2} \sqrt{\frac{\operatorname{Var}\left[\Delta X_{k} \mid \mathcal{F}_{k-1}\right]}{E\left[X_{k}^{2} \mid \mathcal{F}_{k-1}\right]}}=\frac{\delta}{2} \sqrt{\frac{\operatorname{Var}[\varrho]}{E\left[(1+\varrho)^{2}\right]}}
$$

for some $\delta<1$.

Example. Suppose that $1+\varrho_{k}$ is lognormally distributed with parameters $b h$ and $\sigma^{2} h$, i.e.,

$$
1+\varrho_{k}=\exp \left(b h+\sigma \sqrt{h} Z_{k}\right)
$$

for i.i.d. standard normal random variables $Z_{k}$. This corresponds to a discretization of the well-known Black-Scholes model of geometric Brownian motion,

$$
S_{t}=S_{0} \exp \left(b t+\sigma W_{t}\right)
$$

or

$$
\frac{d S_{t}}{S_{t}}=\left(b+\frac{1}{2} \sigma^{2}\right) d t+\sigma d W_{t}
$$

with a discretization step size of $h$ : we simply take $X_{k}=S_{k h}$. If $S$ has growth rate $\mu$ and the instantaneous interest rate is $r$ in continuous time, $b$ is given by $b=\mu-r-\frac{1}{2} \sigma^{2}$. For this example, we obtain

$$
c_{\mathrm{MVT}}(0)=\frac{\left(e^{\left(b+\frac{1}{2} \sigma^{2}\right) h}-1\right)^{2}}{e^{\left(2 b+\sigma^{2}\right) h}\left(e^{\sigma^{2} h}-1\right)}=\frac{\left(b+\frac{1}{2} \sigma^{2}\right)^{2}}{\sigma^{2}} h+O\left(h^{2}\right)
$$


and

$$
\frac{1}{c_{\mathrm{SR}}}=e^{\left(2 b+2 \sigma^{2}\right) h}-2 e^{\left(b+\frac{1}{2} \sigma^{2}\right) h}+1=\sigma^{2} h+O\left(h^{2}\right) .
$$

As $h$ tends to 0 , a uniform bound on $c_{\mathrm{MVT}}(0)$ corresponds to the boundedness of the squared market price of risk

$$
\frac{\left(b+\frac{1}{2} \sigma^{2}\right)^{2}}{\sigma^{2}}=\left(\frac{\mu-r}{\sigma}\right)^{2} .
$$

This is a familiar condition from other work on more general continuous-time models with random coefficients $\mu, r, \sigma$. On the other hand, $c_{\mathrm{SR}}$ explodes as $h$ tends to 0 which seems to bode ill for a continuous-time version of the present approach. However, a moment's thought reveals that things are not as bad as they may appear. In fact, the condition of substantial risk imposes a lower bound on the returns in the form

$$
E\left[\varrho_{k}^{2} \mid \mathcal{F}_{k-1}\right] \geq \frac{1}{c_{\mathrm{SR}}}
$$

and the natural continuous-time analogue of this assumption is that the time derivative of the quadratic variation of the return process should be uniformly bounded below. In a diffusion model of the Black-Scholes type, this is simply the familiar condition that the volatility matrix should be uniformly elliptic.

Finally, let us look at the condition (2.6) of small transaction costs which states that

$$
2 \lambda \sqrt{\frac{E\left[X_{k}^{2} \mid \mathcal{F}_{k-1}\right]}{\operatorname{Var}\left[\Delta X_{k} \mid \mathcal{F}_{k-1}\right]}}=2 \lambda \sqrt{\frac{e^{\left(2 b+2 \sigma^{2}\right) h}}{e^{\left(2 b+\sigma^{2}\right) h}\left(e^{\sigma^{2} h}-1\right)}}
$$

should be of the order $O(1)$. Squaring out and comparing with (2.6) shows that this requires

$$
\lambda \leq \delta \frac{\sigma}{2} \sqrt{h}+O(h)
$$

for some $\delta<1$ so that the transaction cost parameter $\lambda$ should be of the order $\sqrt{h}$. This is in perfect agreement with all known asymptotic results on option pricing under transaction costs; see for instance Henrotte (1993), Lott (1993), Kusuoka (1995), Kabanov/Safarian (1997) or Ahn/Dayal/Grannan/Swindle (1995). This ends the example.

Remark. As pointed out by the referee, typical market conditions $(\lambda=.05 \%$ and $\sigma=15 \%)$ lead to a minimum step size of the order of one hour which is perfectly reasonable. For less liquid markets, however, (2.6) may be a genuine restriction.

We now return to our general model with i.i.d. returns and consider the contingent claim

$$
\bar{\vartheta}_{T+1}=0 \quad, \quad \bar{\eta}_{T}=\left|X_{T}\right|^{\beta}
$$

for some $\beta>0$. (Although $X$ is nonnegative, we write absolute values to avoid confusion with the processes $X^{\gamma}$ for $\gamma \in \Gamma$.) Proposition 2 tells us that to compute $\vartheta_{T}^{*}$, we have to minimize

$$
\operatorname{Var}\left[V_{T}\left(\varphi^{*}\right)-\vartheta_{T} \Delta X_{T}+\lambda X_{T}\left|\vartheta_{T+1}^{*}-\vartheta_{T}\right| \mid \mathcal{F}_{T-1}\right]
$$


with respect to $\vartheta_{T}$. Since the function $c \mapsto c-\lambda|c|$ is a bijection for $0 \leq \lambda<1$ and since $V_{T}\left(\varphi^{*}\right)=\left|X_{T}\right|^{\beta}$ and $\vartheta_{T+1}^{*}=\bar{\vartheta}_{T+1}=0$, we obtain

$$
\vartheta_{T}^{*}-\lambda\left|\vartheta_{T}^{*}\right|=\frac{\operatorname{Cov}\left(V_{T}\left(\varphi^{*}\right), X_{T} \mid \mathcal{F}_{T-1}\right)}{\operatorname{Var}\left[X_{T} \mid \mathcal{F}_{T-1}\right]}=\left|X_{T-1}\right|^{\beta-1} \frac{\operatorname{Cov}\left((1+\varrho)^{\beta}, \varrho\right)}{\operatorname{Var}[\varrho]} \geq 0,
$$

because $\operatorname{Cov}\left(Y^{\beta}, Y\right) \geq 0$ for $\beta>0$. This implies that

$$
\vartheta_{T}^{*}=\frac{1}{1-\lambda}\left|X_{T-1}\right|^{\beta-1} \frac{\operatorname{Cov}\left((1+\varrho)^{\beta}, \varrho\right)}{\operatorname{Var}[\varrho]}=b\left|X_{T-1}\right|^{\beta-1}
$$

with

$$
b:=\frac{1}{1-\lambda} \frac{\operatorname{Cov}\left((1+\varrho)^{\beta}, \varrho\right)}{\operatorname{Var}[\varrho]} \geq 0 .
$$

From the martingale property of $C\left(\varphi^{*}\right)$, the optimal value at date $T-1$ is then given by

$$
\begin{aligned}
V_{T-1}\left(\varphi^{*}\right) & =E\left[V_{T}\left(\varphi^{*}\right)-\vartheta_{T}^{*} \Delta X_{T}+\lambda X_{T}\left|\Delta \vartheta_{T+1}^{*}\right| \mid \mathcal{F}_{T-1}\right] \\
& =E\left[\left|X_{T}\right|^{\beta}-b\left|X_{T-1}\right|^{\beta-1} X_{T-1} \varrho_{T}+\lambda X_{T-1}\left(1+\varrho_{T}\right) b\left|X_{T-1}\right|^{\beta-1} \mid \mathcal{F}_{T-1}\right] \\
& =\left|X_{T-1}\right|^{\beta} E\left[(1+\varrho)^{\beta}-b \varrho+\lambda b(1+\varrho)\right] \\
& =c\left|X_{T-1}\right|^{\beta}
\end{aligned}
$$

with

$$
c:=E\left[(1+\varrho)^{\beta}\right]+b(\lambda-(1-\lambda) E[\varrho]) .
$$

Let us now assume that $\varrho$ has a continuous distribution. The same then holds for $\vartheta_{T}^{*}=b \prod_{j=1}^{T-1}\left(1+\varrho_{j}\right)^{\beta-1}$, and so

$$
I_{\left\{\vartheta_{T}^{*}=\vartheta_{T-1}\right\}}=0 \quad P \text {-a.s. for every } \mathcal{F}_{T-2} \text {-measurable random variable } \vartheta_{T-1} \text {. }
$$

By Proposition 2, the optimal $\vartheta_{T-1}^{*}$ minimizes

$$
\operatorname{Var}\left[V_{T-1}\left(\varphi^{*}\right)-\vartheta_{T-1} \Delta X_{T-1}+\lambda X_{T-1}\left|\vartheta_{T}^{*}-\vartheta_{T-1}\right| \mid \mathcal{F}_{T-2}\right]
$$

with respect to $\vartheta_{T-1}$ and satisfies

$$
\vartheta_{T-1}^{*}=\frac{\operatorname{Cov}\left(V_{T-1}\left(\varphi^{*}\right)+\lambda X_{T-1} \vartheta_{T}^{*} \operatorname{sign}\left(\Delta \vartheta_{T}^{*}\right), X_{T-1}\left(1+\lambda \operatorname{sign}\left(\Delta \vartheta_{T}^{*}\right)\right) \mid \mathcal{F}_{T-2}\right)}{\operatorname{Var}\left[X_{T-1}\left(1+\lambda \operatorname{sign}\left(\Delta \vartheta_{T}^{*}\right)\right) \mid \mathcal{F}_{T-2}\right]}
$$

according to Theorem 6 and (4.7). Plugging in (4.5) and (4.6) for $\vartheta_{T}^{*}$ and $V_{T-1}\left(\varphi^{*}\right)$ therefore shows that $\vartheta_{T-1}^{*}$ is a solution of the implicit equation

$$
\vartheta_{T-1}^{*}=F\left(\vartheta_{T-1}^{*},\left|X_{T-2}\right|^{\beta-1}\right),
$$

where the function $F$ is defined by

$$
F(t, z):=z \frac{\operatorname{Cov}\left((1+\varrho)^{\beta}(c+\lambda b f(t, z, \varrho)),(1+\varrho)(1+\lambda f(t, z, \varrho))\right)}{\operatorname{Var}[(1+\varrho)(1+\lambda f(t, z, \varrho))]}
$$


with

$$
f(t, z, \varrho):=\operatorname{sign}\left(b z(1+\varrho)^{\beta-1}-t\right) .
$$

It would now be very pleasant to have $\vartheta_{T}^{*}$ and $\vartheta_{T-1}^{*}$ of opposite signs because this would force the signs in (4.8) and (4.10) to always equal 1. Since $\vartheta_{T}^{*} \geq 0 P$-a.s., let us suppose for a moment that $\vartheta_{T-1}^{*} \leq 0 P$-a.s. This implies that

$$
\begin{aligned}
\vartheta_{T-1}^{*} & =\frac{\operatorname{Cov}\left(c\left|X_{T-1}\right|^{\beta}+\lambda b\left|X_{T-1}\right|^{\beta}, X_{T-1}(1+\lambda) \mid \mathcal{F}_{T-2}\right)}{\operatorname{Var}\left[X_{T-1}(1+\lambda) \mid \mathcal{F}_{T-2}\right]} \\
& =\left|X_{T-2}\right|^{\beta-1} \frac{c+\lambda b}{1+\lambda} \frac{\operatorname{Cov}\left((1+\varrho)^{\beta}, 1+\varrho\right)}{\operatorname{Var}[1+\varrho]}
\end{aligned}
$$

and so we see that we then must have

$$
c+\lambda b \leq 0
$$

However,

$$
c+\lambda b=E\left[(1+\varrho)^{\beta}\right]+2 \lambda b-b(1-\lambda) E[\varrho]
$$

will not always be nonpositive; a simple counter-example is the case where $E[\varrho]=0$ which is here equivalent to saying that $X$ is a martingale. In general, the sign of $\vartheta_{T}^{*}-\vartheta_{T-1}^{*}$ will therefore be random, and this unfortunately makes the analysis of $F$ and its fixed point very complicated.

Example (continued). Consider again the case where $1+\varrho_{k}$ is lognormally distributed with parameters $b h$ and $\sigma^{2} h$ as in (4.4). Then we can compute the function $F$ in (4.9) more explicitly since it reduces to various combinations of expressions of the form

$$
E\left[(1+\varrho)^{\alpha} I_{\left\{b z(1+\varrho)^{\beta-1}-t>0\right\}}\right]=: h_{>}(\alpha, t, z) .
$$

and

$$
E\left[(1+\varrho)^{\alpha} I_{\left\{b z(1+\varrho)^{\beta-1}-t<0\right\}}\right]=: h_{<}(\alpha, t, z) .
$$

An elementary calculation with the lognormal distribution yields

$$
h_{>}(\alpha, t, z)= \begin{cases}e^{b \alpha+\frac{1}{2} \alpha^{2} \sigma^{2}} \Phi\left(\frac{b+\alpha \sigma^{2}-\frac{1}{\beta-1} \log \frac{t}{b z}}{\sigma}\right) & \text { for } \beta>1 \\ e^{b \alpha+\frac{1}{2} \alpha^{2} \sigma^{2}} \Phi\left(\frac{\frac{1}{\beta-1} \log \frac{t}{b z}-b-\alpha \sigma^{2}}{\sigma}\right) & \text { for } \beta<1\end{cases}
$$

and

$$
h_{<}(\alpha, t, z)=1-h_{>}(\alpha, t, z),
$$

where $\Phi$ is as usual the distribution function of the standard normal distribution. Thus we can express $F(t, z)$ in explicit form with the help of the functions $h_{>}(\alpha, t, z)$ and $h_{<}(\alpha, t, z)$ for suitable values of $\alpha$. However, the result is still fairly complicated and does not provide much help for the determination of $\vartheta_{T-1}^{*}$. It seems at present that the only way to find an optimal strategy is to compute the fixed point of $F$ numerically and to proceed from there. 


\section{Appendix: An abstract conditional optimization problem}

This appendix contains a general technical result which is used in the proof of our main result in section 3 . Let $(\Omega, \mathcal{F}, P)$ be a probability space and $\mathcal{G} \subseteq \mathcal{F}$ a sub- $\sigma$-algebra of $\mathcal{F}$. Let $U, Y, Z$ be $\mathcal{F}$-measurable real-valued random variables satisfying $U \in \mathcal{L}^{2}(P), Z \in \mathcal{L}^{2}(P)$ and $Y Z \in \mathcal{L}^{2}(P)$, and let $\lambda \in[0,1)$ be a fixed real number. We consider the conditional variance

$$
f(c, \omega):=\operatorname{Var}[U-c Z+\lambda Z|Y-c| \mid \mathcal{G}](\omega)
$$

and want to show that it is minimized almost surely at some $c^{*}(\omega)$ which is $\mathcal{G}$-measurable and can be described more explicitly with the help of the first order conditions for optimality. Since $U, Y, Z$ are real random variables, there exists a regular conditional distribution for $(U, Y, Z)$ given $\mathcal{G}$. In the sequel, all conditional expectations, variances and covariances given $\mathcal{G}$ which involve $U, Y$ and $Z$ will be computed with respect to this regular conditional distribution.

Lemma A1. For P-almost every $\omega, c \mapsto f(c, \omega)$ is a continuous function with left- and right-hand derivatives $f_{-}^{\prime}(c, \omega)$ and $f_{+}^{\prime}(c, \omega)$ given by

$$
f_{-}^{\prime}(c, \omega)=-2 \operatorname{Cov}(U-c Z+\lambda Z|Y-c|, Z(1+\lambda \overline{\operatorname{sign}}(Y-c)) \mid \mathcal{G})(\omega)
$$

and

$$
f_{+}^{\prime}(c, \omega)=-2 \operatorname{Cov}(U-c Z+\lambda Z|Y-c|, Z(1+\lambda \underline{\operatorname{sign}}(Y-c)) \mid \mathcal{G})(\omega)
$$

respectively, where $\overline{\text { sign }}$ and sign are defined by (3.4).

Proof. The continuity of $f$ in $c$ is obvious, and by symmetry, it is enough to prove the formula for $f_{+}^{\prime}$. From the definition of $f$, we obtain

$$
\begin{aligned}
& \frac{f(c+h, \omega)-f(c, \omega)}{h} \\
& =\operatorname{Cov}\left(-Z+\lambda Z \frac{|Y-c-h|-|Y-c|}{h}, 2 U-2 c Z-h Z+\lambda Z(|Y-c-h|+|Y-c|) \mid \mathcal{G}\right)(\omega)
\end{aligned}
$$

for any $h>0$. Since

$$
\lim _{h \searrow 0} \frac{|Y-c-h|-|Y-c|}{h}=-\underline{\operatorname{sign}}(Y-c) \quad P \text {-a.s. }
$$

and

$$
|| Y-c-h|-| Y-c|| \leq h,
$$

the dominated convergence theorem yields

$$
\begin{aligned}
f_{+}^{\prime}(c, \omega) & =\lim _{h \searrow 0} \frac{f(c+h, \omega)-f(c, \omega)}{h} \\
& =\operatorname{Cov}(-Z-\lambda Z \underline{\operatorname{sign}}(Y-c), 2 U-2 c Z+2 \lambda Z|Y-c| \mid \mathcal{G})(\omega)
\end{aligned}
$$

which proves the assertion.

q.e.d. 
The next result establishes the existence of a $\mathcal{G}$-measurable minimizer $c^{*}$.

Proposition A2. Assume that $\operatorname{Var}[Z \mid \mathcal{G}]>0$ P-a.s. Then there exists a $\mathcal{G}$-measurable random variable $c^{*}$ such that for $P$-almost every $\omega$,

$$
f\left(c^{*}(\omega), \omega\right) \leq f(c, \omega) \quad \text { for all } c \in \mathbb{R}
$$

Proof. We first show that

$$
\lim _{|c| \rightarrow \infty} f(c, \omega)=+\infty \quad P \text {-a.s. }
$$

In fact, writing $|Y-c|=(Y-c) \operatorname{sign}(Y-c)$ yields

$$
\begin{aligned}
f(c, \omega)= & c^{2} \operatorname{Var}[Z+\lambda Z \operatorname{sign}(Y-c) \mid \mathcal{G}](\omega) \\
& -2 c \operatorname{Cov}(Z(1+\lambda \operatorname{sign}(Y-c)), U+\lambda Y Z \operatorname{sign}(Y-c) \mid \mathcal{G})(\omega) \\
& +\operatorname{Var}[U+\lambda Y Z \operatorname{sign}(Y-c) \mid \mathcal{G}](\omega) \\
= & : c^{2} h_{1}(c, \omega)-2 c h_{2}(c, \omega)+h_{3}(c, \omega) .
\end{aligned}
$$

For fixed $\omega, h_{2}(c, \omega)$ and $h_{3}(c, \omega)$ are both bounded as functions of $c$ due to the integrability assumptions on $U, Y, Z$. Moreover,

$$
\begin{aligned}
\lim _{c \rightarrow \pm \infty} h_{1}(c, \omega) & =\lim _{c \rightarrow \pm \infty} \operatorname{Var}[Z(1+\lambda \operatorname{sign}(Y-c)) \mid \mathcal{G}](\omega) \\
& =\operatorname{Var}[Z(1 \mp \lambda) \mid \mathcal{G}](\omega) \\
& =(1 \mp \lambda)^{2} \operatorname{Var}[Z \mid \mathcal{G}](\omega)>0 \quad P \text {-a.s. }
\end{aligned}
$$

by dominated convergence, and this implies (A.1). Due to the continuity of $f(c, \omega)$ in $c$, we conclude that for $P$-almost all $\omega, c \mapsto f(c, \omega)$ admits a minimum. In order to construct a $\mathcal{G}$-measurable minimizer, let $D_{n}:=\left\{j 2^{-n} \mid j \in \mathbb{Z}\right\}$ be the set of dyadic rationals of order $n$ and define

$$
c_{n}(\omega):=\inf \left\{c \in D_{n} \mid f(c, \omega) \leq f\left(c^{\prime}, \omega\right) \text { for all } c^{\prime} \in D_{n}\right\} .
$$

Since $\omega \mapsto f(c, \omega)$ is $\mathcal{G}$-measurable for fixed $c$, the random variable $c_{n}$ is clearly $\mathcal{G}$-measurable. (A.1) implies that $\left(c_{n}(\omega)\right)_{n \in \mathbb{N}}$ is bounded in $n$ for $P$-almost every $\omega$, and from the continuity of $f(c, \omega)$ in $c$, we conclude that $c^{*}:=\liminf _{n \rightarrow \infty} c_{n}$ has all the desired properties.

q.e.d.

In order to describe $c^{*}$ with the help of the first order conditions for optimality, we now introduce the function $g$ on $\mathbb{R} \times[0,1] \times \Omega$ defined by

$$
g(c, \alpha, \omega):=\operatorname{Cov}\left(U+\lambda Y Z S^{\alpha, c}, Z\left(1+\lambda S^{\alpha, c}\right) \mid \mathcal{G}\right)(\omega)-c \operatorname{Var}\left[Z\left(1+\lambda S^{\alpha, c}\right) \mid \mathcal{G}\right](\omega)
$$

with

$$
S^{\alpha, c}:=\alpha \overline{\operatorname{sign}}(Y-c)+(1-\alpha) \underline{\operatorname{sign}}(Y-c) .
$$

Observe that

$$
f_{+}^{\prime}(c, \omega)=-2 g(c, 0, \omega)
$$


and

$$
f_{-}^{\prime}(c, \omega)=-2 g(c, 1, \omega)
$$

by Lemma A1.

Proposition A3. Assume that $\operatorname{Var}[Z \mid \mathcal{G}]>0$ P-a.s. and let $c^{*}$ be given as in Proposition A2. Then there exists a $\mathcal{G}$-measurable random variable $\alpha^{*}$ with values in $[0,1]$ such that

$$
g\left(c^{*}(\omega), \alpha^{*}(\omega), \omega\right)=0 \quad \text { for P-almost every } \omega .
$$

Proof. Since $c^{*}(\omega)$ minimizes $c \mapsto f(c, \omega)$ for $P$-almost every $\omega$, we have

$$
f_{-}^{\prime}\left(c^{*}(\omega), \omega\right) \leq 0 \leq f_{+}^{\prime}\left(c^{*}(\omega), \omega\right) \quad P \text {-a.s. }
$$

hence

$$
g\left(c^{*}(\omega), 0, \omega\right) \leq 0 \leq g\left(c^{*}(\omega), 1, \omega\right) \quad P \text {-a.s. }
$$

But $\omega \mapsto g\left(c^{*}(\omega), \alpha, \omega\right)$ is $\mathcal{G}$-measurable for every fixed $\alpha$ and $\alpha \mapsto g\left(c^{*}(\omega), \alpha, \omega\right)$ is $P$ a.s. continuous, and so the existence of $\alpha^{*}$ follows from the subsequent lemma.

q.e.d.

Lemma A4. Let $G$ be a real-valued function on $[0,1] \times \Omega$ satisfying

$$
\omega \mapsto G(\alpha, \omega) \text { is } \mathcal{G} \text {-measurable for all } \alpha \in[0,1]
$$

and

$$
\begin{aligned}
& \text { For } P \text {-almost every } \omega \in \Omega, \alpha \mapsto G(\alpha, \omega) \text { is continuous and satisfies } \\
& G(0, \omega) G(1, \omega) \leq 0 \text {. }
\end{aligned}
$$

Then there exists a $\mathcal{G}$-measurable random variable $\alpha^{*}$ with values in $[0,1]$ such that

$$
G\left(\alpha^{*}(\omega), \omega\right)=0 \quad \text { for P-almost all } \omega .
$$

Proof. (A.3) implies that for $P$-almost every $\omega, \alpha \mapsto G(\alpha, \omega)$ has a zero in $[0,1]$ so that

$$
\min _{\alpha \in[0,1]}|G(\alpha, \omega)|=0 \quad P \text {-a.s. }
$$

Thanks to (A.2), the existence of a $\mathcal{G}$-measurable minimizer can thus be proved by virtually the same argument as in the proof of Proposition A2.

q.e.d.

\section{References}

H. Ahn, M. Dayal, E. Grannan and G. Swindle (1995), "Option Replication with Transaction Costs: General Diffusion Limits", preprint, University of California at Santa Barbara

B. Bensaid, J.-P. Lesne, H. Pagès and J. Scheinkman (1992), "Derivative Asset Pricing with Transaction Costs", Mathematical Finance 2, 63-86 
P. P. Boyle and T. Vorst (1992), "Option Replication in Discrete Time with Transaction Costs", Journal of Finance 47, 271-293

G. M. Constantinides and T. Zariphopoulou (1996), "Universal Bounds on Option Prices with Proportional Transaction Costs", preprint, University of Chicago

J. Cvitanić and I. Karatzas (1996), "Hedging and Portfolio Optimization under Transaction Costs: A Martingale Approach", Mathematical Finance 6, 133-165

M. H. A. Davis, V. G. Panas and T. Zariphopoulou (1993), "European Option Pricing with Transaction Costs", SIAM Journal on Control and Optimization 31, 470-493

F. Delbaen, P. Monat, W. Schachermayer, M. Schweizer and C. Stricker (1997), "Weighted Norm Inequalities and Hedging in Incomplete Markets", Finance and Stochastics 1, 181-227

C. Edirisinghe, V. Naik and R. Uppal (1993), "Optimal Replication of Options with Transactions Costs and Trading Restrictions", Journal of Financial and Quantitative Analysis $28,117-138$

P. Henrotte (1993), "Transactions Costs and Duplication Strategies", preprint, Graduate School of Business, Stanford University

S. D. Hodges and A. Neuberger (1989), "Optimal Replication of Contingent Claims Under Transactions Costs", The Review of Futures Markets 8, 222-239

E. Jouini and H. Kallal (1995), "Martingales and Arbitrage in Securities Markets with Transaction Costs", Journal of Economic Theory 66, 178-197

Yu. M. Kabanov and M. M. Safarian (1997), "On Leland's Strategy of Option Pricing with Transactions Costs", Finance and Stochastics 1, 239-250

P.-F. Koehl, H. Pham and N. Touzi (1996), "Option Pricing under Transaction Costs: A Martingale Approach", preprint, CREST, Paris

S. Kusuoka (1995), "Limit Theorem on Option Replication Cost with Transaction Costs", Annals of Applied Probability 5, 198-221

H. E. Leland (1985), "Option Pricing and Replication with Transactions Costs", Journal of Finance 40, 1283-1301

K. Lott (1993), "Ein Verfahren zur Replikation von Optionen unter Transaktionskosten in stetiger Zeit", Ph.D. thesis, Universität der Bundeswehr München

F. Mercurio and T. C. F. Vorst (1997), "Option Pricing and Hedging in Discrete Time with Transaction Costs and Incomplete Markets", in: M. A. H. Dempster and S. R. Pliska (eds.), 'Mathematics of Derivative Securities', Cambridge University Press, 190-215

R. C. Merton (1989), "On the Application of the Continuous-Time Theory of Finance to Financial Intermediation and Insurance", The Geneva Papers on Risk and Insurance 14, $225-261$

F. Ortu (1996), "Arbitrage and Martingales in Securities Markets with Transaction Costs: A Linear Programming Approach", working paper 7/1996, University of Trieste

J.-L. Prigent (1995), "Incomplete Markets: Convergence of Options Values under the Minimal Martingale Measure", preprint, University of Cergy-Pontoise 
W. J. Runggaldier and M. Schweizer (1995), "Convergence of Option Values under Incompleteness", in: E. Bolthausen, M. Dozzi and F. Russo (eds.), "Seminar on Stochastic Analysis, Random Fields and Applications", Birkhäuser, 365-384

M. Schäl (1994), "On Quadratic Cost Criteria for Option Hedging”, Mathematics of Operations Research 19, 121-131

M. Schweizer (1988), "Hedging of Options in a General Semimartingale Model", Diss. ETH Zürich 8615

M. Schweizer (1995), "Variance-Optimal Hedging in Discrete Time", Mathematics of Operations Research 20, 1-32

Q. Shen (1991), "Bid-Ask Prices For Call Options With Transaction Costs", preprint, University of Michigan

H. Shirakawa and H. Konno (1995), "Pricing of Options under the Proportional Transaction Costs", preprint 95-12, Tokyo Institute of Technology

H. M. Soner, S. E. Shreve and J. Cvitanić (1995), "There is No Nontrivial Hedging Portfolio for Option Pricing with Transaction Costs", Annals of Applied Probability 5, 327355 